\title{
ON CONVEX SETS THAT MINIMIZE THE AVERAGE DISTANCE
}

\author{
Antoine LEMENANT $^{1}$ And EdoARdo MAInini ${ }^{2}$
}

\begin{abstract}
In this paper we study the compact and convex sets $K \subseteq \Omega \subseteq \mathbb{R}^{2}$ that minimize

$$
\int_{\Omega} \operatorname{dist}(\mathbf{x}, K) \mathrm{d} \mathbf{x}+\lambda_{1} \operatorname{Vol}(K)+\lambda_{2} \operatorname{Per}(K)
$$

for some constants $\lambda_{1}$ and $\lambda_{2}$, that could possibly be zero. We compute in particular the second order derivative of the functional and use it to exclude smooth points of positive curvature for the problem with volume constraint. The problem with perimeter constraint behaves differently since polygons are never minimizers. Finally using a purely geometrical argument from Tilli [J. Convex Anal. 17 (2010) 583-595] we can prove that any arbitrary convex set can be a minimizer when both perimeter and volume constraints are considered.
\end{abstract}

Mathematics Subject Classification. 49Q10, 49K30.

Received September 7, 2010.

Published online 16 January 2012.

\section{INTRODUCTION}

Given a constant $\ell>0$ and a measure $\mu$ on a domain $\Omega \subseteq \mathbb{R}^{N}$, the classical irrigation problem considered in $[6-9,14,15,18,19,21,22]$ consists in minimizing

$$
\mathcal{F}(\Sigma):=\int_{\Omega} \operatorname{dist}(\mathbf{x}, \Sigma) \mathrm{d} \mu(\mathbf{x})
$$

over all the compact and connected sets $\Sigma$ such that $\mathcal{H}^{1}(\Sigma) \leq \ell$. Here $\operatorname{dist}(\mathbf{x}, \Sigma):=\inf \{|\mathbf{x}-\mathbf{y}|: \mathbf{y} \in \Sigma\}$. Whereas the topological configuration [6] and the blow up limits [19] are now well understood, the optimal regularity for the minimizer remains an open question. In particular it is still not known whether a minimizer could admit or not a point at which the blow up limit is a corner (we call them "corner points", see [19]). It is worth mentioning that the possible existence of such corner points is the crucial fact that makes any regularity result difficult to obtain (see $[14,15])$.

\footnotetext{
Keywords and phrases. Shape optimization, distance functional, optimality conditions, convex analysis, second order variation, gamma-convergence.

1 Université Paris Diderot - Paris 7, U.F.R de Mathématiques, Site Chevaleret, Case 7012, 75205 Paris Cedex 13, France. lemenant@ann.jussieu.fr

2 Dipartimento di Matematica 'F. Casorati', Universià degli Studi di Pavia, via Ferrata 1, 27100 Pavia, Italy. edoardo.mainini@unipv.it
} 
In this paper we concentrate on a slightly different but related problem. Instead of minimizing among onedimensional sets $\Sigma$ (that traditionally represent an irrigation network), we minimize here among solid bodies $K$, that are moreover convex. More precisely, given some nonnegative constants $\lambda_{1}, \lambda_{2}$, a domain $\Omega \subseteq \mathbb{R}^{2}$ and a positive measure $\mu$ (for most of our investigation, $\mu$ is the Lebesgue measure $\mathcal{L}^{2}$ ), we consider the class of admissible sets

$$
\mathcal{A}:=\{K \subseteq \bar{\Omega}, K \text { compact and convex }\}
$$

and the problem

$$
\min _{K \in \mathcal{A}} \mathcal{F}(K)+\lambda_{1} \operatorname{Vol}(K)+\lambda_{2} \operatorname{Per}(K)
$$

where $\mathcal{F}$ is still the average distance functional defined in (1.1). Here $\operatorname{Per}(K)$ means the perimeter in the sense of De Giorgi when $K$ has non empty interior (and in this case $\operatorname{Per}(K)=\operatorname{Per}(\operatorname{Int}(K))$ ). Now if $K$ has empty interior, then it is a segment (because it is convex) and we let $\operatorname{Per}(K)=2 \mathcal{H}^{1}(K)$ in this case.

Here $\lambda_{1}$ or $\lambda_{2}$ could be 0 (but not both of them), which let us the freedom to choose the constraint that we want to study. We are particulary interested by the problem with volume constraint. For this problem the convexity constraint on $K$ is crucial to hope to obtain non trivial minimizers, and we are able to exclude smooth points of positive curvature (Thm. 4.2). The proof uses the second order derivative of the average distance functional $\mathcal{F}$, which itself relies on the second derivative of the distance function along smooth vector fields stated here as a general lemma (Lem. 3.3) and which is interesting on its own. In particular it seems that our functional satisfies some concavity properties as studied in [3].

We would like to emphasize that according to our knowledge, this second derivative (i.e. Lem. 3.3) is new and may be interesting for other purposes, like for the classical average distance problem. This is probably one of the main results of this paper. It can be seen for instance as a complement of [9], where the first order is computed.

The problem becomes different if we assume an additional perimeter constraint (i.e. minimizing with both perimeter and volume constraints). As we shall see later, in this case one can argue as Tilli [22] to prove that any bounded convex domain is a minimizer (Thm. 2.7). This is interesting enough because it provides existence of minimizers for the average distance functional, with appropriate constraints, that actually admit corner points. As was said before, this fact remains an open question for the original irrigation problem mentioned in the first paragraph above.

The main motivation for studying problem (1.2) is principally theoretical. Our goal is to learn more about the properties of the average distance functional and its minimizers. On the other hand akin to the irrigation problem, it might not be difficult to find some applications of problem (1.2). A possible interpretation could be that $K$ represents an artificial lake, that a mayor wants to dig in his city. The surface of the lake is prescribed and for some reasons, purely esthetic say, he wants this lake to be convex. Then the mayor wants this lake to be the closest to everyone in average in his town, according to the density of population. Therefore, to find the optimal shape of the lake he needs to solve problem (1.2).

In Section 6 we approximate the functional by $\Gamma$-convergence in view of numerical computations (Thm. 6.4). However, the convexity constraint makes those computations difficult to implement in practice with a computer. For this convergence we use the well known fact that the distance functional is the limit as $p \rightarrow+\infty$ of the $p$-compliance functional as it was shown in [5]. This fact is also theoretically interesting because it emphasis the link between a purely geometric problem involving the distance functional with an analytic problem involving the $p$-Laplace operator.

Furthermore, the analogue problem replacing the average distance by the $p$-compliance functional is also interesting to consider for its own and has never been studied. 


\section{First ElEMENTARY FACTS}

\subsection{Existence of minimizers}

We first prove the existence of minimizers.

Proposition 2.1. For any bounded domain $\Omega \subseteq \mathbb{R}^{2}$ and positive measure $\mu$, problem (1.2) admits a solution.

Proof. Consider a minimizing sequence $K_{n} \in \mathcal{A}$ of admissible sets such that

$$
\lim _{n \rightarrow+\infty} \mathcal{F}\left(K_{n}\right)+\lambda_{1} \operatorname{Vol}\left(K_{n}\right)+\lambda_{2} \operatorname{Per}\left(K_{n}\right)=\inf _{K \in \mathcal{A}} \mathcal{F}(K)+\lambda_{1} \operatorname{Vol}(K)+\lambda_{2} \operatorname{Per}(K)
$$

Since $\Omega$ is bounded, according to Blaschke theorem we can assume that, up to a subsequence (not relabelled), $K_{n}$ converges to a compact set $K \subseteq \bar{\Omega}$ for the Hausdorff distance. It is then very classical (see e.g. [4]), using the convexity of $K_{n}$, that $K \mapsto \operatorname{Per}(K)$ and $K \mapsto \operatorname{Vol}(K)$ are semicontinuous with respect to this convergence, and this holds true even if $K$ has an empty interior. Then the Hausdorff convergence ensures that dist $\left(x, K_{n}\right)$ converges to $\operatorname{dist}(x, K)$ pointwise and since $x \mapsto \operatorname{dist}\left(x, K_{n}\right)$ are Lipschitz with uniform constant we deduce that $\operatorname{dist}\left(x, K_{n}\right)$ converges to $\operatorname{dist}(x, K)$ uniformly in $x$. Thus passing to the limit in $\int_{\Omega} \operatorname{dist}\left(x, K_{n}\right) \mathrm{d} \mu$ we have that $K$ is a minimizer. Finally, we have to prove that $K$ is convex. If $K$ is empty or reduced to one point, then it is convex and we have nothing to prove. Assume now that $K$ contains at least two points $x$ and $y$. Let $x_{n}$ and $y_{n}$ belong to $K_{n}$ and be the closest points to $x$ and $y\left(i . e . \operatorname{dist}\left(x, K_{n}\right)=\operatorname{dist}\left(x, x_{n}\right)\right.$ and $\left.\operatorname{dist}\left(y, K_{n}\right)=\operatorname{dist}\left(y, y_{n}\right)\right)$. Since $K_{n}$ is convex it holds $\left[x_{n}, y_{n}\right] \subset K_{n}$. And since $K_{n}$ converges to $K$ for the Hausdorff distance, we have in particular that $\operatorname{dist}\left(x, x_{n}\right)=\operatorname{dist}\left(x, K_{n}\right) \leq d_{H}\left(K, K_{n}\right) \rightarrow 0$ and the same for $y$. In other words $x_{n}$ converges to $x$ and $y_{n}$ converges to $y$. But this implies that the segment $\left[x_{n}, y_{n}\right]$ converges to the segment $[x, y]$ for the Hausdorff distance, and since $K$ is the Hausdorff limit of the $K_{n}$, the segment $[x, y]$ must belong to $K$ and this implies that $K$ is convex.

Remark 2.2 (triviality without convexity constraint for the problem penalized by volume). Observe that in the case $\lambda_{1}>0$ and $\lambda_{2}=0$ (volume penalization only), if we remove the convexity constraint, then the infimum of the average distance is zero, and is achieved taking a sequence of sets composed by a union of more and more disjoints balls of infinitesimal radius that spread almost everywhere (commonly referred as "homogenization").

The following interesting remark was communicated to us by Jimmy Lamboley, and says that the convexity constraint can be removed for the problem penalized by the perimeter. It shows in particular that the problems with volume or perimeter penalization are two substantially different problems.

Remark 2.3 (uselessness of convexity constraint for the problem penalized by the perimeter in dimension 2 ). If $N=2$ then it is well known that $\operatorname{Per}(C(K)) \leq \operatorname{Per}(K)$, where $C(K)$ is the convex hull of $K$. On the other hand it is clear that $\mathcal{F}(C(K)) \leq \mathcal{F}(K)$. Therefore, the problem $\min \left\{\mathcal{F}(K)+\lambda_{2} \operatorname{Per}(K), K \subseteq\right.$ $\bar{\Omega}, K$ compact and conncected $\}$ is equivalent to the problem $\min \left\{\mathcal{F}(K)+\lambda_{2} \operatorname{Per}(K), K \subseteq \bar{\Omega}, K \in \mathcal{A}\right\}$, when $\Omega$ is convex.

\subsection{Minimizing among disks}

Let $\Omega$ be convex, let $\mathbf{x}_{0} \in \Omega$ and let $f(\theta), \theta \in[0,2 \pi)$, be the polar representation of $\partial \Omega$ with respect to $\mathbf{x}_{0}$. Consider the problem of finding the best disk $B_{r}\left(\mathbf{x}_{0}\right)$ centered at $\mathbf{x}_{0}$ for the functional $\mathcal{F}\left(B_{r}\left(\mathbf{x}_{0}\right)\right)+$ $\lambda_{1} \operatorname{Vol}\left(B_{r}\left(\mathbf{x}_{0}\right)\right), r \in\left[0, \operatorname{dist}\left(\mathbf{x}_{0}, \partial \Omega\right)\right]$. Notice that in this case

$$
\begin{aligned}
\int_{\Omega} \operatorname{dist}\left(\mathbf{x}, B_{r}\left(\mathbf{x}_{0}\right)\right) \mathrm{d} \mathbf{x}+\lambda_{1} \operatorname{Vol}\left(B_{r}\left(\mathbf{x}_{0}\right)\right) & =\int_{0}^{2 \pi} \int_{r}^{f(\theta)}(\rho-r) \rho \mathrm{d} \rho \mathrm{d} \theta+\lambda_{1} \pi r^{2} \\
& =\frac{1}{3} \int_{0}^{2 \pi} f(\theta)^{3} \mathrm{~d} \theta-\frac{1}{2} r \int_{0}^{2 \pi} f(\theta)^{2} \mathrm{~d} \theta+\frac{\pi}{3} r^{3}+\lambda_{1} \pi r^{2} .
\end{aligned}
$$


Hence,

$$
\begin{aligned}
\frac{\mathrm{d}}{\mathrm{d} r}\left(\int_{\Omega} \operatorname{dist}\left(\mathbf{x}, B_{r}\left(\mathbf{x}_{0}\right)\right) \mathrm{d} \mathbf{x}+\lambda_{1} \operatorname{Vol}\left(B_{r}\left(\mathbf{x}_{0}\right)\right)\right) & =-\frac{1}{2} \int_{0}^{2 \pi} f(\theta)^{2} \mathrm{~d} \theta+\pi r^{2}+2 \lambda_{1} \pi r, \\
\frac{\mathrm{d}^{2}}{\mathrm{~d} r^{2}}\left(\int_{\Omega} \operatorname{dist}\left(\mathbf{x}, B_{r}\left(\mathbf{x}_{0}\right)\right) \mathrm{d} \mathbf{x}+\lambda_{1} \operatorname{Vol}\left(B_{r}\left(\mathbf{x}_{0}\right)\right)\right) & =2 \pi\left(\lambda_{1}+r\right) .
\end{aligned}
$$

The optimal radius is therefore

$$
\bar{r}=-\lambda_{1}+\frac{1}{\pi}\left(\pi^{2} \lambda_{1}^{2}+\frac{\pi}{2} \int_{0}^{2 \pi} f(\theta)^{2} \mathrm{~d} \theta\right)^{1 / 2} .
$$

For instance, if $\Omega=B_{1}\left(\mathbf{x}_{0}\right)$ we see that

$$
\bar{r}=\sqrt{\lambda_{1}^{2}+1}-\lambda_{1} .
$$

As a consequence, $\bar{r}=1$ for $\lambda_{1}=0$ (obvious, without constraints the optimal set is $\Omega$ itself), and when $\lambda_{1}$ is increased, $\bar{r}$ decreases to 0 . Larger values of $\lambda_{1}$ mean that we search for smaller volume sets.

Remark 2.4. After the more general analysis of the next sections, we will actually be able to say that a circle is never a minimizer of $\mathcal{F}(\cdot)+\lambda_{1} \operatorname{Vol}(\cdot)$, independently of the shape of $\Omega$. Some heuristics in this direction are also contained in the next proposition.

\subsection{Breaking the symmetry}

The following intuitive argument was suggested by Buttazzo, and seems to say that the minimizers with volume constraint tend to break the symmetry of the problem in order to decrease the average distance functional. The idea is to consider minimizers with infinitesimal volume.

Proposition 2.5. Let $\mu=\mathcal{L}^{2}$ and $\lambda_{2}=0$ (that is, we consider the problem with only volume penalization). Let $\Omega:=B(0,1)$ be the unit ball in $\mathbb{R}^{2}$. Then a long segment has better average distance than a small concentric ball.

Proof. Intuitively the proposition is quite clear, but let us check it with explicit computations. Consider first the convex set $K:=B(0, \varepsilon)$ for a small $\varepsilon$. Then

$$
\begin{aligned}
\int_{B(0,1)} \operatorname{dist}(\mathbf{x}, K) \mathrm{d} \mathbf{x} & =\int_{0}^{2 \pi} \int_{\varepsilon}^{1}(r-\varepsilon) r \mathrm{~d} r \mathrm{~d} \theta \\
& =2 \pi\left(\left[\frac{r^{3}}{3}-\varepsilon \frac{r^{2}}{2}\right]_{\varepsilon}^{1}\right) \\
& =\frac{2 \pi}{3}-\pi \varepsilon+o(\varepsilon) .
\end{aligned}
$$

Now we take as a competitor the diameter $S:=[-1,1] \times\{0\}$ of zero volume. Then a simple computation yields

$$
\int_{B(0,1)} \operatorname{dist}(\mathbf{x}, S) \mathrm{d} \mathbf{x}=4 \int_{0}^{1} \int_{0}^{\sqrt{1-x^{2}}} y \mathrm{~d} y \mathrm{~d} x=\frac{4}{3} .
$$

Since for $\varepsilon$ small $\frac{4}{3}<\frac{2 \pi}{3}-\pi \varepsilon+o(\varepsilon)$, we see that the segment does much better than a small ball, even with strictly less volume.

Remark 2.6. Notice that this phenomenon is less obvious while minimizing with perimeter constraint. This is why the problem with only volume constraint is preferred by the authors than the one with only perimeter constraint, because of the tendency to break the symmetry of $\Omega$ which is quite intriguing. 


\subsection{Convex sets as minimizers with both perimeter and volume constraints}

Here we prove using an argument from Tilli [22] that under both perimeter and volume constraint, any convex set is a minimizer.

Theorem 2.7. Let $\mu=\mathcal{L}^{2}$. Let $K_{0} \subseteq \mathbb{R}^{2}$ be a convex set, with $\operatorname{Per}\left(K_{0}\right)=\ell$ and $\operatorname{Vol}\left(K_{0}\right)=V$. Then for any $T>0, K_{0}$ is the minimizer for the average distance functional $\mathcal{F}$ in the class of convex sets $K$ such that $\operatorname{Per}(K)=\ell, \operatorname{Vol}(K)=V$, in the particular domain $\Omega=\Omega_{T}:=\left\{\operatorname{dist}\left(x, K_{0}\right)<T\right\}$.

Proof. We recall that for every convex set $K \subseteq \mathbb{R}^{2}$ the following standard equality holds

$$
\mathscr{L}^{2}(\{0<\operatorname{dist}(\mathbf{x}, K) \leq t\})=\operatorname{Per}(K) t+\pi t^{2} .
$$

The equality (2.1) is well known and usually referred as the Steiner-Minkowski formula (see e.g. Thm. 3.2.35 p. 271 of [12]). A way to prove it is to establish (2.1) first when $K$ is a polygon and then approximate a convex set uniformly by a sequence of polygons and pass to the limit.

Let now $K \subseteq \Omega_{T}$ be a convex set such that $\operatorname{Per}(K)=\ell$ and $\operatorname{Vol}(K)=V$. Let also $D_{T}:=\operatorname{diam}\left(\Omega_{T}\right)$. Reasoning as Tilli (see [22]) in his proof of minimality of $C^{1,1}$ curves for the classic average distance problem, we make use of the slicing formula and obtain

$$
\begin{aligned}
\int_{\Omega_{T}} \operatorname{dist}(\mathbf{x}, K) \mathrm{d} \mathbf{x} & =\int_{0}^{D_{T}} \mathcal{L}^{2}\left(\left\{\mathbf{x} \in \Omega_{T} \backslash K: \operatorname{dist}(\mathbf{x}, K)>s\right\}\right) \mathrm{d} s \\
& =\mathcal{L}^{2}\left(\Omega_{T} \backslash K\right) D_{T}-\int_{0}^{D_{T}} \mathcal{L}^{2}\left(\left\{\mathbf{x} \in \Omega_{T}: 0<\operatorname{dist}(\mathbf{x}, K) \leq s\right\}\right) \mathrm{d} s \\
& \geq\left(\mathcal{L}^{2}\left(\Omega_{T}\right)-V\right) D_{T}-\int_{0}^{D_{T}} \min \left\{\mathcal{L}^{2}\left(\Omega_{T} \backslash K\right), \ell s+\pi s^{2}\right\} \mathrm{d} s
\end{aligned}
$$

Here we exploited (2.1) together with the obvious inequality

$$
\mathcal{L}^{2}\left(\left(\Omega_{T} \backslash K\right) \cap(\{0<\operatorname{dist}(x, K) \leq s\})\right) \leq \min \left\{\mathcal{L}^{2}\left(\Omega_{T} \backslash K\right), \mathcal{L}^{2}(\{0<\operatorname{dist}(x, K) \leq s\})\right\},
$$

holding for any $s \geq 0$. But this inequality is an equality, for any $s$, if and only if $K=K_{0}$, since $\Omega_{T}$ is the neighborhood of $K_{0}$. Then, as $\operatorname{Per}\left(K_{0}\right)=\ell$ and $\operatorname{Vol}\left(K_{0}\right)=V$, for $K=K_{0}$ we have equality also in $(2.2)$ and the proof is concluded.

Remark 2.8. Notice that when $K_{0}$ is a ball, then no other convex competitor but this ball has same volume and perimeter. However, when $K_{0}$ is not a ball we believe that the class of convex sets with exactly same perimeter and volume is large enough to bring some interest in Theorem 2.7.

Remark 2.9. The link with problem (1.2) is that $\lambda_{1}$ and $\lambda_{2}$ should be the appropriate Lagrange multipliers associated with the problem of Theorem 2.7 where we minimize over the class of convex sets with restricted perimeter and area. On the other hand a rigorous proof of the equivalence between the two problems (restricted one and penalized one) is not available at the moment and would need further work.

\section{HighER ORDER ANALYSIS}

In this section we are going to perform a second order expansion of the average distance along smooth vector fields. We prefer to develop this argument in a more general setting, considering average distance from a compact connected $\mathcal{H}^{1}$-rectifiable set $\Sigma$, not necessarily the boundary of a convex set. In Section 4 we will give an application to problem (1.2). 
It is necessary to introduce some more notation. Let $\Sigma$ be as above. Given a point $\mathbf{x} \in \Omega$, we denote by $\pi^{\Sigma}(\mathbf{x})$ its projection on the set $\Sigma$, so that

$$
\left|\mathbf{x}-\pi^{\Sigma}(\mathbf{x})\right|=\operatorname{dist}(\mathbf{x}, \Sigma) .
$$

Of course, there can be points where the projection map $\pi^{\Sigma}$ is not single-valued. These correspond to points of non differentiability of the distance function from $\Sigma$. The set of these points is called the Ridge set of $\Sigma$ and is denoted by $\mathfrak{R}_{\Sigma}$. Hence

$$
\mathfrak{R}_{\Sigma}:=\left\{\mathbf{x} \in \Omega: \sharp\left(\pi^{\Sigma}(\mathbf{x})\right)>1\right\} .
$$

It is well known that if $\Sigma$ is the boundary of a convex set $K$, there is $\mathfrak{R}_{\Sigma} \subseteq K$ (the set $K$ has "infinite reach", in the terminology of [11]). We recall that the map $\mathbf{x} \mapsto \operatorname{dist}(\mathbf{x}, \Sigma)$ is $C^{1}$ over $\Omega \backslash\left(\Sigma \cup \overline{\Re_{\Sigma}}\right)$, see for instance [16], Proposition 3.6. If $\Sigma$ is a $C^{1,1}$ curve, then there exists the 'tubular neighborhood' $N$, where all the points have a unique projection on $\Sigma$. In this case we have the $C^{1}$ property above in $N \backslash K$, so that the distance function is regular if we are not too far from $\Sigma$. More precisely, the function $\mathbf{x} \mapsto \operatorname{dist}^{2}(\mathbf{x}, \Sigma)$ is $C^{r}$ in $\Omega \backslash \overline{\Re_{\Sigma}}$ as soon as $\Sigma$ is $C^{r}, r \geq 2$ (see [16], Prop. 4.8). We also refer to [10] for a general discussion about the regularity of the distance function, with respect to the regularity of $\Sigma$.

Let $\Phi_{\varepsilon}: \mathbb{R}^{2} \rightarrow \mathbb{R}^{2}$ denote the one parameter group of diffeomorphisms defined by

$$
\Phi_{\varepsilon}:=\operatorname{Id}+\varepsilon \mathbf{X},
$$

where $\mathbf{X} \in C_{0}^{\infty}\left(\mathbb{R}^{2} ; \mathbb{R}^{2}\right)$. Let $\Sigma_{\varepsilon}:=\Phi_{\varepsilon}(\Sigma)$. Our goal is to approximate, given $\mathbf{x} \in \Omega$, the quantity

$$
\operatorname{dist}\left(\mathbf{x}, \Sigma_{\varepsilon}\right)
$$

at the different orders with respect to $\varepsilon$.

The first order term is already known (see $[1,9]$ ). Indeed, there is

$$
\left.\frac{\mathrm{d}}{\mathrm{d} \varepsilon} \operatorname{dist}\left(\mathbf{x}, \Sigma_{\varepsilon}\right)\right|_{\varepsilon=0}=\left\langle\nabla \operatorname{dist}(\mathbf{x}, \Sigma), \mathbf{X}\left(\pi^{\Sigma}(\mathbf{x})\right)\right\rangle=\left\langle\frac{\pi^{\Sigma}(\mathbf{x})-\mathbf{x}}{\left|\pi^{\Sigma}(\mathbf{x})-\mathbf{x}\right|}, \mathbf{X}\left(\pi^{\Sigma}(\mathbf{x})\right)\right\rangle .
$$

This formula does not make sense for any $\mathbf{x}$, since $\nabla \operatorname{dist}(\mathbf{x}, \Sigma)$ is well defined only if $\mathbf{x}$ does not belong to $\Sigma \cup \mathfrak{R}_{\Sigma}$. But the distance function is Lipschitz, so that $\nabla$ dist defines a $L^{\infty}$ function. Therefore we can also state

$$
\left.\frac{\mathrm{d}}{\mathrm{d} \varepsilon}\left[\int_{\Omega} \operatorname{dist}\left(\mathbf{x}, \Sigma_{\varepsilon}\right) \mathrm{d} \mu(\mathbf{x})\right]\right|_{\varepsilon=0}=\int_{\Omega}\left\langle\nabla \operatorname{dist}(\mathbf{x}, \Sigma), \mathbf{X}\left(\pi^{\Sigma}(\mathbf{x})\right)\right\rangle \mathrm{d} \mu(\mathbf{x}) .
$$

The integral is well defined as soon as $\mu$ does not charge $\Sigma$ and its Ridge set (of course the Lebesgue measure will work). We stress that the above formula holds for any closed connected set $\Sigma$, as shown in [9].

Remark 3.1. When performing the second order approximation, we will need tangent and normal vectors to $\Sigma$. Therefore, it seems not possible to avoid a $C^{2}$ regularity assumption for $\Sigma$. One could possibly work in the $C^{1,1}$ case, with right and left curvatures. But even for smooth curves, it is well known that the second derivatives of $\mathbf{x} \mapsto \operatorname{dist}(\mathbf{x}, \Sigma)$ are in general not bounded, the problem arising again because of the Ridge set. A sufficient condition for our computations to make sense will be $\mathbf{x} \notin \overline{\mathfrak{R}_{\Sigma}}$. But we stress that, when taking the second derivative in $\varepsilon$ along the diffeomorphism (3.1), outside $\overline{\mathfrak{R}_{\Sigma}}$, in principle we expect to find an unbounded function. For a $C^{3}$ curve, it is shown in [16], Section 4, that $\overline{\mathfrak{R}_{\Sigma}}$ is a $\mathcal{H}^{1}$-rectifiable set, but the same property is not known for the $C^{2}$ case. In [16] it is also shown that the closure of the Ridge set can have positive $\mathcal{L}^{2}$ measure in the $C^{1,1}$ case. These remarks show that, unlike the case of the first derivative (3.2), in general it will not be possible to integrate the second order derivative $\frac{\mathrm{d}^{2}}{\mathrm{~d} \varepsilon^{2}} \operatorname{dist}\left(\mathbf{x}, \Sigma_{\varepsilon}\right)$ at $\varepsilon=0$ over $\Omega$, even in the case $\mu=\mathcal{L}^{2}$. By the way, this problem does not happen when $\Sigma=\partial K$, where $K$ is a convex set, and we integrate on $\Omega \backslash K$, as in (1.1). Indeed, the infinite-reach property ensures that dist is more regular outside $K$. Analogously, in the 
$C^{2}$ case one could integrate $\frac{\mathrm{d}^{2}}{\mathrm{~d} \varepsilon^{2}} \operatorname{dist}\left(\mathbf{x}, \Sigma_{\varepsilon}\right)$ at $\varepsilon=0$ against the Lebesgue measure over a small neighborhood of $\Sigma$, since the tubular neighborhood property ensures that the second derivatives are bounded therein. Indeed, by the regularity in the tubular neighborhood, it is enough to take a smaller neighborhood to have bounded second derivatives.

Let us begin the computations assuming that $\Sigma$ is a smooth curve. We denote its arc-length parametrization as $t \in[-L, L] \mapsto \mathbf{f}(t), L>0$. This way one can write $\mathbf{f}(t)=\mathbf{f}(0)+t \mathbf{f}_{1}(0)+t^{2} \mathbf{f}_{2}(0)+o\left(t^{2}\right)$, where $\mathbf{f}_{1}$ is the tangent unit vector and $\mathbf{f}_{2}$ is normal and directed to the center of curvature. The set $\Sigma_{\varepsilon}$ is itself a smooth curve whose parametrization $\mathbf{f}_{\varepsilon}(t)$ is given as $\Phi_{\varepsilon}(\mathbf{f}(t)), t \in[-L, L]$. Now, consider a point $\mathbf{x} \in \Omega$ such that $\mathbf{x} \notin\left(\Sigma \cup \overline{\mathfrak{R}_{\Sigma}}\right)$. We have

$$
\operatorname{dist}^{2}\left(\mathbf{x}, \Sigma_{\varepsilon}\right)=\inf \left\{|\mathbf{z}-\mathbf{x}|^{2}: \mathbf{z} \in \Sigma_{\varepsilon}\right\}=\inf \left\{\left|\mathbf{x}-\mathbf{f}_{\varepsilon}(t)\right|^{2}: t \in[-L, L]\right\} .
$$

If $\bar{t} \in[-L, L]$ is a point where the minimum is achieved, then the circle with radius $\operatorname{dist}\left(\mathbf{x}, \Sigma_{\varepsilon}\right)$, centered at $\mathbf{x}$, is tangent to the curve $\Sigma_{\varepsilon}$ itself. Hence, if $\partial_{t} \mathbf{f}_{\varepsilon}(\bar{t})$ represents the tangent vector of $\mathbf{f}_{\varepsilon}(t)$ at the point $\bar{t}$, there is

$$
\left\langle\left(\mathbf{x}-\mathbf{f}_{\varepsilon}(\bar{t})\right), \partial_{t} \mathbf{f}_{\varepsilon}(\bar{t})\right\rangle=0 .
$$

Since we are searching for a Taylor approximation of $\operatorname{dist}(\mathbf{x}, \Sigma)$ in terms of $\varepsilon$, we search for a minimizer $\bar{t}=\bar{t}_{\varepsilon}$ of the form

$$
\bar{t}_{\varepsilon}=\sum_{n=0}^{+\infty} t_{n} \varepsilon^{n} .
$$

Suppose, without loss of generality, that $\mathbf{f}(0)=\pi^{\Sigma}(\mathbf{x})$, and let $\mathbf{x}_{0}$ denote this point. Notice that at the order zero, the solution of equation (3.4) is of course $t=0$, since the point satisfying that orthogonality condition is $\mathbf{x}_{0}$ itself. We immediately deduce $t_{0}=0$, so that we can let the sum in (3.5) start from $n=1$. For the computation, we need also the expansions of $\mathbf{f}$ and $\mathbf{X}$. We have

$$
\begin{aligned}
\mathbf{f}(t) & =\sum_{i=0}^{+\infty} \mathbf{f}_{i} t^{i}, \quad t \in[-L, L], \\
\mathbf{X}(\mathbf{y}) & =\sum_{j=0}^{+\infty} \mathbf{X}_{j} \otimes\left(\mathbf{y}-\mathbf{x}_{0}\right)^{\otimes j}, \quad \mathbf{y} \in \Omega,
\end{aligned}
$$

where $\otimes$ denotes the suitable order tensorial product and $(\cdot)^{\otimes j}$ represents the $j$-th power with respect to such product. Notice that our conventions give $\mathbf{f}_{0}=\mathbf{x}_{0}$, hence composing the two expansions we have

$$
\mathbf{X}(\mathbf{f}(t))=\sum_{j=0}^{+\infty} \mathbf{X}_{j} \otimes\left(\sum_{i=1}^{+\infty} \mathbf{f}_{i} t^{i}\right)^{\otimes j}
$$

which we conveniently rewrite as a simple series as

$$
\sum_{k=0}^{+\infty} \mathbf{g}_{k} t^{k}
$$

Here, each coefficient $\mathbf{g}_{k}$ is a vector which can be written as a finite sum involving the $\mathbf{f}_{i}$ 's and the $\mathbf{X}_{j}$ 's, and whose expression might be found by induction. For instance we have

$$
\begin{aligned}
& \mathbf{g}_{0}=\mathbf{X}_{0}=\mathbf{X}\left(\pi^{\Sigma}(\mathbf{x})\right) \\
& \mathbf{g}_{1}=\mathbf{X}_{1} \otimes \mathbf{f}_{1}=\nabla \mathbf{X}\left(\pi^{\Sigma}(\mathbf{x})\right) \otimes \mathbf{f}^{\prime}(0), \\
& \mathbf{g}_{2}=\mathbf{X}_{1} \otimes \mathbf{f}_{2}+\mathbf{X}_{2} \otimes \mathbf{f}_{1}^{\otimes 2}=\frac{1}{2} \nabla \mathbf{X}\left(\pi^{\Sigma}(\mathbf{x})\right) \otimes \mathbf{f}^{\prime \prime}(0)+\frac{1}{2} \nabla^{2} \mathbf{X}\left(\pi^{\Sigma}(\mathbf{x})\right) \otimes\left(\mathbf{f}^{\prime}(0)\right)^{\otimes 2}
\end{aligned}
$$


Hence we get

$$
\begin{aligned}
\mathbf{f}_{\varepsilon}(t) & =\mathbf{f}(t)+\varepsilon \mathbf{X}(\mathbf{f}(t))=\sum_{i=0}^{+\infty}\left(\mathbf{f}_{i}+\varepsilon \mathbf{g}_{i}\right) t^{i}, \\
\partial_{t} \mathbf{f}_{\varepsilon}(t) & =\sum_{j=1}^{+\infty} j\left(\mathbf{f}_{j}+\varepsilon \mathbf{g}_{j}\right) t^{j-1} .
\end{aligned}
$$

Let us now write the desired Taylor expansion. Taking advantage of (3.3) and (3.8), we have

$$
\operatorname{dist}^{2}\left(\mathbf{x}, \Sigma_{\varepsilon}\right)=\left|\mathbf{x}-\mathbf{f}_{\varepsilon}\left(\bar{t}_{\varepsilon}\right)\right|^{2}=\left|\mathbf{x}-\sum_{i=0}^{+\infty}\left(\mathbf{f}_{i}+\varepsilon \mathbf{g}_{i}\right)\left(\sum_{n=0}^{+\infty} t_{n} \varepsilon^{n}\right)^{i}\right|^{2} .
$$

We write explicitly the form of the second order approximation. From the relation above we deduce

$$
\begin{aligned}
\operatorname{dist}^{2}\left(\mathbf{x}, \Sigma_{\varepsilon}\right)= & \left|\mathbf{x}-\mathbf{f}_{0}-\mathbf{f}_{1}\left(t_{1} \varepsilon+t_{2} \varepsilon^{2}+o\left(\varepsilon^{2}\right)\right)-\mathbf{f}_{2}\left(t_{1}^{2} \varepsilon^{2}+o\left(\varepsilon^{2}\right)\right)-\varepsilon\left(\mathbf{g}_{0}+t_{1} \mathbf{g}_{1} \varepsilon\right)+o\left(\varepsilon^{2}\right)\right|^{2} \\
= & \left|\mathbf{x}-\mathbf{f}_{0}\right|^{2}-2\left\langle\mathbf{x}-\mathbf{f}_{0}, t_{1} \mathbf{f}_{1}+\mathbf{g}_{0}\right\rangle \varepsilon \\
& +\left|t_{1} \mathbf{f}_{1}+\mathbf{g}_{0}\right|^{2} \varepsilon^{2}-2\left\langle\mathbf{x}-\mathbf{f}_{0}, t_{2} \mathbf{f}_{1}+t_{1}^{2} \mathbf{f}_{2}+t_{1} \mathbf{g}_{1}\right\rangle \varepsilon^{2}+o(\varepsilon)^{2} .
\end{aligned}
$$

Since $\mathbf{f}_{0}=\mathbf{x}_{0}$ by convention, and since $\mathbf{f}_{1}$ is the tangent unit vector to $\mathbf{f}(t)$ at the point $t=0$, it is clear that the scalar product $\left\langle\mathbf{x}-\mathbf{f}_{0}, \mathbf{f}_{1}\right\rangle$ vanishes (this is the 0 -th order condition). We are left with

$$
\begin{aligned}
\operatorname{dist}^{2}\left(\mathbf{x}, \Sigma_{\varepsilon}\right)= & \left|\mathbf{x}-\mathbf{f}_{0}\right|^{2}-2\left\langle\mathbf{x}-\mathbf{f}_{0}, \mathbf{g}_{0}\right\rangle+\left|t_{1} \mathbf{f}_{1}+\mathbf{g}_{0}\right|^{2} \varepsilon^{2}-2\left\langle\mathbf{x}-\mathbf{f}_{0}, t_{1}^{2} \mathbf{f}_{2}+t_{1} \mathbf{g}_{1}\right\rangle \varepsilon^{2}+o(\varepsilon)^{2} \\
= & \left|\mathbf{x}-\pi^{\Sigma}(\mathbf{x})\right|^{2}-2\left\langle\mathbf{x}-\pi^{\Sigma}(\mathbf{x}), \mathbf{X}\left(\pi^{\Sigma}(\mathbf{x})\right)\right\rangle \varepsilon \\
& -2\left\langle\mathbf{x}-\pi^{\Sigma}(\mathbf{x}), \frac{1}{2} t_{1}^{2} \mathbf{f}^{\prime \prime}(0)+t_{1} \nabla \mathbf{X}\left(\pi^{\Sigma}(\mathbf{x})\right) \otimes \mathbf{f}^{\prime}(0)\right\rangle \varepsilon^{2} \\
& +\left|t_{1} \mathbf{f}^{\prime}(0)+\mathbf{X}\left(\pi^{\Sigma}(\mathbf{x})\right)\right|^{2} \varepsilon^{2}+o\left(\varepsilon^{2}\right) .
\end{aligned}
$$

We pass to the square root, and making use of the elementary relation

$$
\sqrt{1+a \varepsilon+b \varepsilon^{2}+o\left(\varepsilon^{2}\right)}=1+\frac{a}{2} \varepsilon+\frac{1}{2}\left(b-\frac{a^{2}}{4}\right) \varepsilon^{2}+o\left(\varepsilon^{2}\right)
$$

we get

$$
\begin{aligned}
\operatorname{dist}\left(\mathbf{x}, \Sigma_{\varepsilon}\right)= & \operatorname{dist}(\mathbf{x}, \Sigma)-\left\langle\mathbf{X}\left(\pi^{\Sigma}(\mathbf{x})\right), \frac{\mathbf{x}-\pi^{\Sigma}(\mathbf{x})}{\left|\mathbf{x}-\pi^{\Sigma}(\mathbf{x})\right|}\right\rangle \varepsilon \\
& -\left\langle\frac{\mathbf{x}-\pi^{\Sigma}(\mathbf{x})}{\left|\mathbf{x}-\pi^{\Sigma}(\mathbf{x})\right|}, \frac{1}{2} t_{1}^{2} \mathbf{f}^{\prime \prime}(0)+t_{1} \nabla \mathbf{X}\left(\pi^{\Sigma}(\mathbf{x})\right) \otimes \mathbf{f}^{\prime}(0)\right\rangle \varepsilon^{2} \\
& +\frac{\left|t_{1} \mathbf{f}^{\prime}(0)+\mathbf{X}\left(\pi^{\Sigma}(\mathbf{x})\right)\right|^{2}}{2\left|\mathbf{x}-\pi^{\Sigma}(\mathbf{x})\right|} \varepsilon^{2}-\frac{1}{2}\left\langle\frac{\mathbf{x}-\pi^{\Sigma}(\mathbf{x})}{\left|\mathbf{x}-\pi^{\Sigma}(\mathbf{x})\right|^{3 / 2}}, \mathbf{X}\left(\pi^{\Sigma}(\mathbf{x})\right)\right\rangle^{2} \varepsilon^{2}+o\left(\varepsilon^{2}\right) .
\end{aligned}
$$

At the first order, we recover the already known approximation $(3.2)$ of $[1,9]$. This shows that $\Phi_{\varepsilon}\left(\pi^{\Sigma}(\mathbf{x})\right)$ is a good approximation of $\pi^{\Sigma_{\varepsilon}}(\mathbf{x})$ at the first order, which is the underlying fact for the arguments therein. As already remarked, the first order approximation does not involve Taylor expansions of $t \mapsto \mathbf{f}(t)$ and $\mathbf{X}$, so that it holds without any regularity assumption. We can now rewrite the optimality condition (3.4) inserting the expansions, and we obtain

$$
\left\langle\left(\mathbf{x}-\sum_{i=0}^{+\infty}\left(\mathbf{f}_{i}+\varepsilon \mathbf{g}_{i}\right) t^{i}\right), \sum_{j=1}^{+\infty} j\left(\mathbf{f}_{j}+\varepsilon \mathbf{g}_{j}\right) t^{j-1}\right\rangle=0 .
$$


The coefficients $t_{n}$ are found using this formula together with (3.5), yielding the condition

$$
\left\langle\left(\mathbf{x}-\sum_{i=0}^{+\infty}\left(\mathbf{f}_{i}+\varepsilon \mathbf{g}_{i}\right)\left(\sum_{n=0}^{+\infty} t_{n} \varepsilon^{n}\right)^{i}\right), \sum_{j=1}^{+\infty} j\left(\mathbf{f}_{j}+\varepsilon \mathbf{g}_{j}\right)\left(\sum_{n=0}^{+\infty} t_{n} \varepsilon^{n}\right)^{j-1}\right\rangle=0 .
$$

One has to compare the coefficients of the different powers of $\varepsilon$. We have already observed that $t_{0}=0$. At the first order, the relation above is

$$
\left\langle\mathbf{x}-\mathbf{f}_{0}, \mathbf{f}_{1}\right\rangle+\left\langle\mathbf{x}-\mathbf{f}_{0}, 2 t_{1} \mathbf{f}_{2}+\mathbf{g}_{1}\right\rangle \varepsilon-\left\langle\mathbf{f}_{1}, t_{1} \mathbf{f}_{1}+\mathbf{g}_{0}\right\rangle \varepsilon+o(\varepsilon)=0 .
$$

We get, using the 0 -th order condition $\left\langle\mathbf{x}-\mathbf{f}_{0}, \mathbf{f}_{1}\right\rangle=0$,

$$
t_{1}=\frac{\left\langle\mathbf{x}-\mathbf{f}_{0}, \mathbf{g}_{1}\right\rangle-\left\langle\mathbf{f}_{1}, \mathbf{g}_{0}\right\rangle}{\left|\mathbf{f}_{1}\right|^{2}-2\left\langle\mathbf{x}-\mathbf{f}_{0}, \mathbf{f}_{2}\right\rangle}=\frac{\left\langle\mathbf{x}-\pi^{\Sigma}(\mathbf{x}), \nabla \mathbf{X}\left(\pi^{\Sigma}(\mathbf{x})\right) \otimes \mathbf{f}^{\prime}(0)\right\rangle-\left\langle\mathbf{f}^{\prime}(0), \mathbf{X}\left(\pi^{\Sigma}(\mathbf{x})\right)\right\rangle}{1-\left\langle\mathbf{x}-\pi^{\Sigma}(\mathbf{x}), \mathbf{f}^{\prime \prime}(0)\right\rangle} .
$$

Now we have to be careful, because the denominator can vanish. With the next proposition, we show that the condition $\mathbf{x} \notin \overline{\Re_{\Sigma}}$ ensures that this is not the case. For the proof, we need to define the 'cut locus' of the set $\Sigma$. We say that $\mathbf{x} \in \Omega$ belongs the cut locus of a $C^{1,1}$ curve $\Sigma$ if there exists $\mathbf{x}_{0} \in \Sigma$ such that the half line from $\mathbf{x}_{0}$ through $\mathbf{x}$ is normal to $\Sigma$ and for $\mathbf{y}$ belonging to the same half line we have $\operatorname{dist}(\mathbf{y}, \Sigma)=\operatorname{dist}\left(\mathbf{y}, \mathbf{x}_{0}\right)$ if and only if $\operatorname{dist}\left(\mathbf{x}, \mathbf{x}_{0}\right) \geq \operatorname{dist}\left(\mathbf{y}, \mathbf{x}_{0}\right)$. In this case, we write $\mathbf{x} \in \mathfrak{C}_{\Sigma}$. The cut locus is a standard object in Riemaniann geometry, we refer to the texts on this subject. Roughly speaking, it is the set of points where geodesics from a set $\Sigma$ minimize 'for the last time' the distance from $\Sigma$. In Euclidean context, minimizing geodesics are normal segments.

Proposition 3.2. Let $\Sigma$ be a $C^{2}$ curve and let $\mathbf{x} \notin \overline{\mathfrak{R}_{\Sigma}}$. Then $\left\langle\mathbf{x}-\pi^{\Sigma}(\mathbf{x}), \mathbf{f}^{\prime \prime}(0)\right\rangle \neq 1$, where $\mathbf{f}$ is the arc-length parametrization of $\Sigma$ as above, such that $\mathbf{f}(0)=\pi^{\Sigma}(\mathbf{x})$.

Proof. First of all, since $\mathbf{x} \notin \mathfrak{R}_{\Sigma}$, the projection $\pi^{\Sigma}(\mathbf{x})=\mathbf{x}_{0}$ is well defined. We have to consider all the points which are projected on $\mathbf{x}_{0}$, which of course lie on the normal line to $\Sigma$ from $\mathbf{x}_{0}$, and to see for which of these points there holds $\left\langle\mathbf{x}-\mathbf{x}_{0}, \mathbf{f}^{\prime \prime}(0)\right\rangle=1$. Indeed, since $\mathbf{f}^{\prime \prime}(0)$ is a normal vector whose length is the curvature at $t=0$, this happens precisely when $\mathbf{x}$ is the center of the osculating circle at $\mathbf{x}_{0}$, which we denote by $\mathbf{x}_{1}$. Let us denote by $\boldsymbol{\nu}$ the unit direction of the half line from $\mathbf{x}_{0}$ through $\mathbf{x}_{1}$. We parametrize it as $\mathbf{x}(s)=\mathbf{x}_{0}+\left(\operatorname{dist}\left(\mathbf{x}_{0}, \mathbf{x}_{1}\right)\right) s \boldsymbol{\nu}$, $s \in[0,+\infty)$, so that indeed $\mathbf{x}(1)=\mathbf{x}_{1}$. It is clear that if $\pi^{\Sigma}(\mathbf{x}(s)) \neq \mathbf{x}_{0}$ for some $s \leq 1$, then $\mathbf{x}_{1}$ itself can not be projected on $\mathbf{x}_{0}$. Hence in this case all the points $\mathbf{x}$ which are projected on $\mathbf{x}_{0}$ have the property

$$
\left\langle\mathbf{x}-\mathbf{x}_{0}, \mathbf{f}^{\prime \prime}(0)\right\rangle \neq 1
$$

It remains to consider the case in which all the points $\mathbf{x}(s), s \leq 1$, are projected on $\mathbf{x}_{0}$. But by the very definition of osculating circle, in this case all the points $\mathbf{x}(s)$ with $s>1$ are no more projected on $\mathbf{x}_{0}$. This means that $\mathbf{x} \in \mathfrak{C}_{\Sigma}$, and it is shown in [16], Proposition 4.8, that for $C^{2}$ curves there holds $\mathfrak{C}_{\Sigma}=\overline{\mathfrak{R}_{\Sigma}}$. This concludes the proof.

With this, we have at our disposal the full expression of the second order approximation of dist $\left(\mathbf{x}, \Sigma_{\varepsilon}\right)$. Since $\mathbf{f}^{\prime}$ and $\mathbf{f}^{\prime \prime}$ appear in (3.10)-(3.11), we can consider a $C^{2}$ curve $\Sigma$.

Lemma 3.3. Let $\mathbf{x} \in \Omega \backslash\left(\Sigma \cup \overline{\Re_{\Sigma}}\right)$ and let $\Sigma$ be a $C^{2}$ curve in a neighborhood of $\pi^{\Sigma}(\mathbf{x})$. Let $\Phi_{\varepsilon}$ be as (3.1) and $\Sigma_{\varepsilon}=\Phi_{\varepsilon}(\Sigma)$. Let moreover $\mathbf{f}$ denote the usual arc-length parametrization of $\Sigma$, with $\mathbf{f}_{0}:=\mathbf{f}(0)=\pi^{\Sigma}(\mathbf{x})=\mathbf{x}_{0}$, $\mathbf{f}_{1}:=\mathbf{f}^{\prime}(0), 2 \mathbf{f}_{2}:=\mathbf{f}^{\prime \prime}(0)$. Then there holds

$$
\operatorname{dist}\left(\mathbf{x}, \Sigma_{\varepsilon}\right)=A_{\Sigma, \mathbf{x}}^{0}+A_{\Sigma, \mathbf{X}}^{1} \varepsilon+A_{\Sigma, \mathbf{X}}^{2} \varepsilon^{2}+o\left(\varepsilon^{2}\right),
$$


where the coefficients are given by

$$
\begin{aligned}
& A_{\Sigma, \mathbf{x}}^{0}=\operatorname{dist}(\mathbf{x}, \Sigma)=\left|\mathbf{x}-\pi^{\Sigma}(\mathbf{x})\right| \\
& A_{\Sigma, \mathbf{x}}^{1}=-\left\langle\mathbf{X}\left(\pi^{\Sigma}(\mathbf{x})\right), \frac{\mathbf{x}-\pi^{\Sigma}(\mathbf{x})}{\left|\mathbf{x}-\pi^{\Sigma}(\mathbf{x})\right|}\right\rangle \\
& A_{\Sigma, \mathbf{X}}^{2}=\frac{\left\langle\mathbf{f}_{1}, \mathbf{X}\left(\pi^{\Sigma}(\mathbf{x})\right)\right\rangle^{2}}{2\left|\mathbf{x}-\pi^{\Sigma}(\mathbf{x})\right|}-\frac{\left(\left\langle\mathbf{x}-\pi^{\Sigma}(\mathbf{x}), \nabla \mathbf{X}\left(\pi^{\Sigma}(\mathbf{x})\right) \otimes \mathbf{f}_{1}\right\rangle-\left\langle\mathbf{f}_{1}, \mathbf{X}\left(\pi^{\Sigma}(\mathbf{x})\right)\right\rangle\right)^{2}}{2\left|\mathbf{x}-\pi^{\Sigma}(\mathbf{x})\right|\left(1-2\left\langle\mathbf{x}-\pi^{\Sigma}(\mathbf{x}), \mathbf{f}_{2}\right\rangle\right)}
\end{aligned}
$$

Proof. We see that $\mathbf{A}_{\Sigma, \mathbf{X}}^{0}, \mathbf{A}_{\Sigma, \mathbf{X}}^{1}$ come directly from (3.10). We have to compute $A_{\Sigma, \mathbf{x}}^{2}$. In the following computations, we keep the more compact notation in terms of $\mathbf{f}_{i}, \mathbf{g}_{i}$ and $\mathbf{x}_{0}$. We also let $\mathbf{X}_{n}$ and $\mathbf{X}_{\tau}$ denote respectively the normal and tangent components of $\mathbf{X}$. Moreover, we use the following obvious facts:

$$
\left\langle\frac{\mathbf{x}-\pi^{\Sigma}(\mathbf{x})}{\left|\mathbf{x}-\pi^{\Sigma}(\mathbf{x})\right|}, \mathbf{X}\left(\pi^{\Sigma}(\mathbf{x})\right)\right\rangle^{2}=\left|\mathbf{X}_{n}\left(\pi^{\Sigma}(\mathbf{x})\right)\right|^{2} \quad \text { and } \quad\left\langle\mathbf{f}_{1}, \mathbf{X}\left(\pi^{\Sigma}(\mathbf{x})\right)\right\rangle^{2}=\left|\mathbf{X}_{\tau}\left(\pi^{\Sigma}(\mathbf{x})\right)\right|^{2}
$$

Let us consider (3.10) and rearrange the second order terms therein. Recall that the expression for the coefficient $t_{1}$ is $(3.11)$. We have

$$
\begin{aligned}
& -\left\langle\frac{\mathbf{x}-\pi^{\Sigma}(\mathbf{x})}{\left|\mathbf{x}-\pi^{\Sigma}(\mathbf{x})\right|}, \frac{1}{2} t_{1}^{2} \mathbf{f}^{\prime \prime}(0)+t_{1} \nabla \mathbf{X}\left(\pi^{\Sigma}(\mathbf{x})\right) \otimes \mathbf{f}^{\prime}(0)\right\rangle \\
& +\frac{\left|t_{1} \mathbf{f}^{\prime}(0)+\mathbf{X}\left(\pi^{\Sigma}(\mathbf{x})\right)\right|^{2}}{2\left|\mathbf{x}-\pi^{\Sigma}(\mathbf{x})\right|}-\frac{1}{2}\left\langle\frac{\mathbf{x}-\pi^{\Sigma}(\mathbf{x})}{\left|\mathbf{x}-\pi^{\Sigma}(\mathbf{x})\right|^{3 / 2}}, \mathbf{X}\left(\pi^{\Sigma}(\mathbf{x})\right)\right\rangle^{2} \\
= & -\left\langle\frac{\mathbf{x}-\mathbf{x}_{0}}{\left|\mathbf{x}-\mathbf{x}_{0}\right|}, t_{1}^{2} \mathbf{f}_{2}+t_{1} \mathbf{g}_{1}\right\rangle+\frac{\left|t_{1} \mathbf{f}_{1}+\mathbf{g}_{0}\right|^{2}}{2\left|\mathbf{x}-\mathbf{x}_{0}\right|}-\frac{1}{2}\left\langle\frac{\mathbf{x}-\mathbf{x}_{0}}{\left|\mathbf{x}-\mathbf{x}_{0}\right|^{3 / 2}}, \mathbf{g}_{0}\right\rangle^{2} \\
= & \frac{1}{\left|\mathbf{x}-\mathbf{x}_{0}\right|}\left[-t_{1}^{2}\left\langle\mathbf{x}-\mathbf{x}_{0}, \mathbf{f}_{2}\right\rangle-t_{1}\left\langle\mathbf{x}-\mathbf{x}_{0}, \mathbf{g}_{1}\right\rangle+\frac{1}{2} t_{1}^{2}+t_{1}\left\langle\mathbf{f}_{1}, \mathbf{g}_{0}\right\rangle+\frac{1}{2}\left|\mathbf{g}_{0}\right|^{2}-\frac{1}{2}\left\langle\frac{\mathbf{x}-\mathbf{x}_{0}}{\left|\mathbf{x}-\mathbf{x}_{0}\right|}, \mathbf{g}_{0}\right\rangle^{2}\right] \\
= & \frac{1}{\left|\mathbf{x}-\mathbf{x}_{0}\right|}\left[\frac{1}{2} t_{1}^{2}\left(1-2\left\langle\mathbf{x}-\mathbf{x}_{0}, \mathbf{f}_{2}\right\rangle\right)-t_{1}\left(\left\langle\mathbf{x}-\mathbf{x}_{0}, \mathbf{g}_{1}\right\rangle-\left\langle\mathbf{f}_{1}, \mathbf{g}_{0}\right\rangle\right)+\frac{1}{2}\left|\mathbf{X}_{\tau}\right|^{2}\right] \\
= & \frac{1}{\left|\mathbf{x}-\mathbf{x}_{0}\right|}\left[\frac{1}{2} \frac{\left(\left\langle\mathbf{x}-\mathbf{x}_{0}, \mathbf{g}_{1}\right\rangle-\left\langle\mathbf{f}_{1}, \mathbf{g}_{0}\right\rangle\right)^{2}}{1-2\left\langle\mathbf{x}-\mathbf{x}_{0}, \mathbf{f}_{2}\right\rangle}-\frac{\left(\left\langle\mathbf{x}-\mathbf{x}_{0}, \mathbf{g}_{1}\right\rangle-\left\langle\mathbf{f}_{1}, \mathbf{g}_{0}\right\rangle\right)^{2}}{1-2\left\langle\mathbf{x}-\mathbf{x}_{0}, \mathbf{f}_{2}\right\rangle}+\frac{1}{2}\left|\mathbf{X}_{\tau}\right|^{2}\right] \\
= & \frac{1}{2\left|\mathbf{x}-\mathbf{x}_{0}\right|}\left[\left\langle\mathbf{f}_{1}, \mathbf{g}_{0}\right\rangle^{2}-\frac{\left(\left\langle\mathbf{x}-\mathbf{x}_{0}, \mathbf{g}_{1}\right\rangle-\left\langle\mathbf{f}_{1}, \mathbf{g}_{0}\right\rangle\right)^{2}}{1-2\left\langle\mathbf{x}-\mathbf{x}_{0}, \mathbf{f}_{2}\right\rangle}\right] .
\end{aligned}
$$

This is the desired expression of the second derivative.

Notice that the second order coefficient in (3.13) could be written also as

$$
A_{\Sigma, \mathbf{x}}^{2}=\frac{1}{2\left|\mathbf{x}-\mathbf{x}_{0}\right|}\left[\frac{-\left\langle\mathbf{x}-\mathbf{x}_{0}, \mathbf{g}_{1}\right\rangle^{2}}{1-2\left\langle\mathbf{x}-\mathbf{x}_{0}, \mathbf{f}_{2}\right\rangle}+\frac{2\left\langle\mathbf{x}-\mathbf{x}_{0}, \mathbf{g}_{1}\right\rangle\left\langle\mathbf{f}_{1}, \mathbf{g}_{0}\right\rangle}{1-2\left\langle\mathbf{x}-\mathbf{x}_{0}, \mathbf{f}_{2}\right\rangle}-\frac{2\left|\mathbf{X}_{\tau}\right|^{2}\left\langle\mathbf{x}-\mathbf{x}_{0}, \mathbf{f}_{2}\right\rangle}{1-2\left\langle\mathbf{x}-\mathbf{x}_{0}, \mathbf{f}_{2}\right\rangle}\right] .
$$

From this formula it is clear that $A_{\Sigma, \mathbf{x}}^{2}$ is finite for $\operatorname{dist}(\mathbf{x}, \Sigma) \rightarrow 0$.

Let us see how this approximation works in some particular situation. First of all, in the case of a normal vector field $\mathbf{X}$ to $\Sigma$, we are left with

$$
A_{\Sigma, \mathbf{X}}^{2}=-\frac{\left\langle\mathbf{x}-\pi^{\Sigma}(\mathbf{x}), \nabla \mathbf{X}\left(\pi^{\Sigma}(\mathbf{x})\right) \otimes \mathbf{f}_{1}\right\rangle^{2}}{2\left|\mathbf{x}-\pi^{\Sigma}(\mathbf{x})\right|\left(1-2\left\langle\mathbf{x}-\pi^{\Sigma}(\mathbf{x}), \mathbf{f}_{2}\right\rangle\right)} .
$$

In the case of a flat curve, $\mathbf{f}_{2}=0$ and we have the following 
Proposition 3.4. Let $\Sigma$ be a closed connected $\mathcal{H}^{1}$-rectifiable set, and let $\Sigma^{F}$ be a flat connected subset of $\Sigma$. Let $\Phi_{\varepsilon}$ be defined as (3.1), with a vector field $\mathbf{X}$ normal to $\Sigma^{F}$. Let $\partial_{\tau}$ denote the derivative in the direction individuated by $\Sigma^{F}$. If $\pi^{\Sigma}(\mathbf{x})$ is in the relative interior of $\Sigma^{F}$, then

$$
\operatorname{dist}\left(\mathbf{x}, \Sigma_{\varepsilon}\right)=\operatorname{dist}(\mathbf{x}, \Sigma)-\left\langle\mathbf{X}\left(\pi^{\Sigma}(\mathbf{x})\right), \frac{\mathbf{x}-\pi^{\Sigma}(\mathbf{x})}{\left|\mathbf{x}-\pi^{\Sigma}(\mathbf{x})\right|}\right\rangle \varepsilon-\frac{1}{2} \operatorname{dist}(\mathbf{x}, \Sigma)\left|\partial_{\tau} \mathbf{X}\left(\pi^{\Sigma}(\mathbf{x})\right)\right|^{2} \varepsilon^{2}+o\left(\varepsilon^{2}\right) .
$$

Proof. We simply need to see how Lemma 3.3 reduces in this case. Let us fix a reference orthonormal basis, with origin in $\pi^{\Sigma}(\mathbf{x})$, an axis parallel to $\Sigma^{F}$ and the other one normal to it, pointing towards the half-plane containing x. $\Sigma$ is $C^{2}$ in a neighborhood of $\pi^{\Sigma}(\mathbf{x})$, since this point is in the relative interior of $\Sigma^{F}$. Let $t \mapsto \mathbf{f}(t)$ be the arclength parametrization of $\Sigma^{F}$. In the reference basis, for the Taylor expansion (3.6) we have $\mathbf{f}_{1}=\mathbf{f}^{\prime}(0)=(1,0)$ and $\mathbf{f}_{2}=\frac{1}{2} \mathbf{f}^{\prime \prime}(0)=(0,0)$. Moreover, since $\mathbf{X}=\left(X_{\tau}, X_{n}\right)$ is normal to $\Sigma^{F}$, there holds

$$
\nabla \mathbf{X}\left(\pi^{\Sigma}(\mathbf{x})\right) \otimes \mathbf{f}^{\prime}(0)=\left(0, \partial_{\tau} X_{n}\left(\pi^{\Sigma}(\mathbf{x})\right)\right)
$$

Hence, from (3.14) we have

$$
A_{\Sigma, \mathbf{x}}^{2}=-\frac{1}{2} \operatorname{dist}(\mathbf{x}, \Sigma)\left[\partial_{\tau} X_{n}\left(\pi^{\Sigma}(\mathbf{x})\right)\right]^{2}
$$

which gives the thesis.

Remark 3.5. Notice that in the case of Proposition 3.4 the denominator in the second order coefficient is distant from 0 . In the regions where this happens, we could integrate the expansion of $\operatorname{dist}(\mathbf{x}, \Sigma)$, for instance with $\mu=\mathcal{L}^{2}$. We stress that this situation occurs also in the exterior of a convex set (recall the discussion in Rem. 3.1). Indeed, if $K$ is convex, $\Sigma=\partial K$ and $\mathbf{x} \in \Omega \backslash K$, the vectors $\mathbf{x}-\pi^{\Sigma}(\mathbf{x})$ and $\mathbf{f}_{2}$ have opposite verse, hence we have

$$
1-2\left\langle\mathbf{x}-\pi^{\Sigma}(\mathbf{x}), \mathbf{f}_{2}\right\rangle \geq 1 .
$$

Then, for any finite measure $\mu$ over $\Omega$, it makes sense to write

$$
\mathcal{G}(K):=\int_{\Omega \backslash K}\left(\frac{\left|\mathbf{X}_{\tau}\left(\pi^{\Sigma}(\mathbf{x})\right)\right|^{2}}{\left|\mathbf{x}-\pi^{\Sigma}(\mathbf{x})\right|}-\frac{\left(\left\langle\mathbf{x}-\pi^{\Sigma}(\mathbf{x}), \nabla \mathbf{X}\left(\pi^{\Sigma}(\mathbf{x})\right) \otimes \mathbf{f}_{1}\right\rangle-\left\langle\mathbf{f}_{1}, \mathbf{X}\left(\pi^{\Sigma}(\mathbf{x})\right)\right\rangle\right)^{2}}{\left|\mathbf{x}-\pi^{\Sigma}(\mathbf{x})\right|\left(1-2\left\langle\mathbf{x}-\pi^{\Sigma}(\mathbf{x}), \mathbf{f}_{2}\right\rangle\right)}\right) \mathrm{d} \mu(\mathbf{x}) .
$$

Here $K_{\varepsilon}=\Phi_{\varepsilon}(K)$, with the usual diffeomorphism (3.1), whereas the unit tangent vector $\mathbf{f}_{1}$ and the normal vector $\mathbf{f}_{2}$ are understood to be computed in correspondence of the point $\pi^{\Sigma}(\mathbf{x})$.

Till the end of this section, we keep the notation of the above remark. $K$ is a convex set, $\Sigma:=\partial K$ has $C^{2}$ regularity and $\mu$ is chosen to be the Lebesgue measure $\mathcal{L}^{2}$. For the description of $\Sigma$, we refer to the notation of Lemma 3.3, with the usual parametrization $t \mapsto \mathbf{f}(t)$. We also let $\mathbf{n}$ denote the unit outward normal, so that with respect to the parametrization there is $\mathbf{n}=\mathbf{n}(t)=-\frac{\mathbf{f}_{2}(t)}{\left|\mathbf{f}_{2}(t)\right|}$. Moreover, $\Sigma_{\varepsilon}:=\partial K_{\varepsilon}$, where $K_{\varepsilon}=\Phi_{\varepsilon}(K)$ and $\Phi_{\varepsilon}$ is given by (3.1). Notice that, since

$$
\mathcal{F}\left(K_{\varepsilon}\right)=\int_{\Omega} \operatorname{dist}\left(\mathbf{x}, K_{\varepsilon}\right) \mathrm{d} \mathbf{x}=\int_{\Omega \backslash K_{\varepsilon}} \operatorname{dist}\left(\mathbf{x}, \Sigma_{\varepsilon}\right),
$$

we may write $\mathcal{F}$ according to the following notation:

$$
\mathcal{F}\left(K_{\varepsilon}\right)=\mathcal{F}_{1}\left(K_{\varepsilon}\right)-\mathcal{F}_{2}\left(K_{\varepsilon}\right),
$$

where

$$
\mathcal{F}_{1}\left(K_{\varepsilon}\right):=\int_{\Omega \backslash K} \operatorname{dist}\left(\mathbf{x}, \Sigma_{\varepsilon}\right) \mathrm{d} \mathbf{x}, \quad \mathcal{F}_{2}\left(K_{\varepsilon}\right):=\int_{K \triangle K_{\varepsilon}} \operatorname{sign}\left\langle\mathbf{X}\left(\pi^{\Sigma}(\mathbf{x})\right), \mathbf{n}\right\rangle \operatorname{dist}\left(\mathbf{x}, \Sigma_{\varepsilon}\right) \mathrm{d} \mathbf{x} .
$$

Here the sign takes into account that the vector field $\mathbf{X}$ might push a point $\mathbf{x} \in \Sigma$ outside or inside $K$, depending on the sign of its scalar product with the outward unit normal at $\mathbf{x}$. 
Proposition 3.6. There holds

$$
\left.\frac{\mathrm{d}}{\mathrm{d} \varepsilon} \mathcal{F}\left(K_{\varepsilon}\right)\right|_{\varepsilon=0}=\left.\frac{\mathrm{d}}{\mathrm{d} \varepsilon} \mathcal{F}_{1}\left(K_{\varepsilon}\right)\right|_{\varepsilon=0}=\int_{\Omega \backslash K}\left\langle\mathbf{X}\left(\pi^{\Sigma}(\mathbf{x})\right), \frac{\pi^{\Sigma}(\mathbf{x})-\mathbf{x}}{\left|\pi^{\Sigma}(\mathbf{x})-\mathbf{x}\right|}\right\rangle \mathrm{d} \mathbf{x} .
$$

Proof. It is clear that $\mathcal{F}_{2}\left(K_{\varepsilon}\right) \rightarrow 0$ as $\varepsilon \rightarrow 0$. We also claim that such term gives no contribution to the first order. Indeed, it is well known (see for instance [13]) that

$$
\operatorname{Vol}\left(K \triangle K_{\varepsilon}\right)=\varepsilon \int_{\Sigma}|\langle\mathbf{X}(\mathbf{x}), \mathbf{n}\rangle| \mathrm{d} \mathcal{H}^{1}(\mathbf{x})+o(\varepsilon),
$$

and since $\operatorname{dist}\left(\mathbf{x}, \Sigma_{\varepsilon}\right) \leq \varepsilon \sup _{\mathbf{x}}|\mathbf{X}(\mathbf{x})|$ over $K \triangle K_{\varepsilon}$, we conclude that

$$
\begin{aligned}
\limsup _{\varepsilon \rightarrow 0} \frac{1}{\varepsilon^{2}}\left|\int_{K \triangle K_{\varepsilon}} \operatorname{sign}\left(\left\langle\mathbf{X}\left(\pi^{\Sigma}(\mathbf{x})\right), \mathbf{n}\right\rangle\right) \operatorname{dist}\left(\mathbf{x}, \Sigma_{\varepsilon}\right) \mathrm{d} \mathbf{x}\right| & \leq \limsup _{\varepsilon \rightarrow 0} \frac{1}{\varepsilon} \sup _{\mathbf{x}}|\mathbf{X}(\mathbf{x})| \operatorname{Vol}\left(K \triangle K_{\varepsilon}\right) \\
& \leq \mathcal{H}^{1}(\Sigma) \sup _{\mathbf{x}}|\mathbf{X}(\mathbf{x})|^{2},
\end{aligned}
$$

so that we are also bounding the second derivative of $\mathcal{F}_{2}\left(K_{\varepsilon}\right)$. As a consequence, at first order we only have the contribution of $\mathcal{F}_{1}$, for which we take the Taylor expansion given by Lemma 3.3 and simply integrate over $\Omega \backslash K$ : after Remark 3.5, we know that the integrals of the first and second order term make sense on $\Omega \backslash K$. The thesis is achieved.

Remark 3.7 (first order necessary conditions). Standard necessary conditions for optimality in problem (1.2) are obtained computing the derivatives of the terms therein. The first derivatives of volume and perimeter under variations along smooth vector fields are well known (see for instance $[9,13]$ ). Together with $(3.18)$, they give the following first order conditions for a minimizer $K \subseteq \Omega$. In the case of perimeter penalization, the set $K$ has to satisfy

$$
\int_{\Omega \backslash K}\left\langle\mathbf{X}\left(\pi^{\Sigma}(\mathbf{x})\right), \frac{\pi^{\Sigma}(\mathbf{x})-\mathbf{x}}{\left|\pi^{\Sigma}(\mathbf{x})-\mathbf{x}\right|}\right\rangle \mathrm{d} \mathbf{x}+\lambda_{2} \int_{\partial K} H\langle\mathbf{X}, \mathbf{n}\rangle \mathrm{d} \mathcal{H}^{1}=0
$$

for any smooth and compactly supported vector field $\mathbf{X}$, where $H$ denotes the mean curvature of $\partial K$. For the case of volume penalization, the strictly convex set $K$ has to satisfy

$$
\int_{\Omega \backslash K}\left\langle\mathbf{X}\left(\pi^{\Sigma}(\mathbf{x})\right), \frac{\pi^{\Sigma}(\mathbf{x})-\mathbf{x}}{\left|\pi^{\Sigma}(\mathbf{x})-\mathbf{x}\right|}\right\rangle \mathrm{d} \mathbf{x}+\lambda_{1} \int_{\partial K}\langle\mathbf{X}, \mathbf{n}\rangle \mathrm{d} \mathcal{H}^{1}=0
$$

for any smooth and compactly supported vector field $\mathbf{X}$. In the latter case, we have to consider the additional convexity constraint, as already discussed (see Rem. 2.3). Hence, in such case we have to restrict to vector fields which keep the set convex. Indeed we can consider any $\mathbf{X}$ if $K$ is a strictly convex set. Otherwise, one has to be careful because if $K$ is convex, but not strictly convex, in general only unilateral variations are allowed, yielding a first order condition with inequality only. We will come back to this issue later in Section 5.

In order to complete the discussion, we end the section with the computation of the second derivative of functional $\mathcal{F}$. For simplicity, and in view of the applications in the subsequent sections, we restrict to the case of normal vector fields $\mathbf{X}$.

Theorem 3.8 (second derivative of $\mathcal{F}$ with respect to a normal variation). Let $\Phi_{\varepsilon}=I d+\varepsilon \mathbf{X}$ with a vector field $\mathbf{X}$ which is assumed to be normal to $\partial K$ (i.e. $\left.\left\langle\mathbf{X}(\mathbf{f}(t)), \mathbf{f}_{1}(t)\right\rangle=0\right)$. Then there holds

$$
\left.\frac{\mathrm{d}^{2}}{\mathrm{~d} \varepsilon^{2}} \mathcal{F}\left(K_{\varepsilon}\right)\right|_{\varepsilon=0}=\mathcal{G}_{n}(K)-\int_{\partial K} \operatorname{sign}\langle\mathbf{X}(\mathbf{x}), \mathbf{n}\rangle|\mathbf{X}(\mathbf{x})|^{2} \mathrm{~d} \mathcal{H}^{1}(\mathbf{x}),
$$


where

$$
\mathcal{G}_{n}(K):=-\int_{\Omega \backslash K} \frac{\left(\left\langle\mathbf{x}-\pi^{\Sigma}(\mathbf{x}), \nabla \mathbf{X}\left(\pi^{\Sigma}(\mathbf{x})\right) \otimes \mathbf{f}_{1}\right\rangle\right)^{2}}{\left|\mathbf{x}-\pi^{\Sigma}(\mathbf{x})\right|\left(1-2\left\langle\mathbf{x}-\pi^{\Sigma}(\mathbf{x}), \mathbf{f}_{2}\right\rangle\right)} \mathrm{d} \mathbf{x} .
$$

Proof. We write as before

$$
\mathcal{F}\left(K_{\varepsilon}\right)=\mathcal{F}_{1}\left(K_{\varepsilon}\right)-\mathcal{F}_{2}\left(K_{\varepsilon}\right)
$$

with $\mathcal{F}_{1}$ and $\mathcal{F}_{2}$ given by (3.17). By the dominated convergence theorem one gets

$$
\frac{\mathrm{d}^{2}}{\mathrm{~d} \varepsilon^{2}} \mathcal{F}_{1}\left(K_{\varepsilon}\right)=\mathcal{G}_{n}(K)
$$

Recall that the definition of $\mathcal{G}$ is (3.16), and here it is reduced to the simpler expression $\mathcal{G}_{n}$ by the fact that $\mathbf{X}$ is a normal vector field.

Now we claim that for all $\mathrm{x} \in K \triangle K_{\varepsilon}$,

$$
\operatorname{dist}\left(\mathbf{x}, \Sigma_{\varepsilon}\right)=\operatorname{dist}\left(\mathbf{x}, \Phi_{\varepsilon}\left(\pi^{\Sigma}(\mathbf{x})\right)\right)+o(\varepsilon) .
$$

To prove the claim notice first that $\operatorname{dist}\left(\mathbf{x}, \Sigma_{\varepsilon}\right) \leq \operatorname{dist}\left(\mathbf{x}, \Phi_{\varepsilon}\left(\pi^{\Sigma}(\mathbf{x})\right)\right)$, thus it is enough to prove the reverse inequality. Let $\mathbf{x}_{\varepsilon}:=\Phi_{\varepsilon}\left(\pi^{\Sigma}(\mathbf{x})\right)$ and $\mathbf{z}_{\varepsilon}:=\pi^{\Sigma_{\varepsilon}}(\mathbf{x})$. By the triangle inequality, we are reduce to prove that $\operatorname{dist}\left(\mathbf{z}_{\varepsilon}, \mathbf{x}_{\varepsilon}\right)=o(\varepsilon)$. But this directly comes from the calculus in the first part of this section. Indeed, using the same notation (see (3.3)-(3.8)), there holds

$$
\mathbf{z}_{\varepsilon}=\mathbf{f}_{\varepsilon}\left(\bar{t}_{\varepsilon}\right)=\mathbf{f}_{0}+\varepsilon \mathbf{g}_{0}+\varepsilon t_{1} \mathbf{f}_{1}+o(\varepsilon)=\mathbf{x}_{\varepsilon}+\varepsilon t_{1} \mathbf{f}_{1}+o(\varepsilon),
$$

where $\mathbf{f}_{1}$ is the unit tangent vector at point $\pi^{\Sigma}(\mathbf{x})$ and $t_{1}$ is given by (3.11). Since $\mathbf{x} \in K \triangle K_{\varepsilon}$, it satisfies

$$
t_{1}=\frac{\left\langle\mathbf{x}-\pi^{\Sigma}(\mathbf{x}), \partial_{\tau} \mathbf{X}\left(\pi^{\Sigma}(\mathbf{x})\right)\right\rangle^{2}}{2\left|\mathbf{x}-\pi^{\Sigma}(\mathbf{x})\right|\left(1-2\left\langle\mathbf{x}-\pi^{\Sigma}(\mathbf{x}), \mathbf{f}_{2}\right\rangle\right.} \leq C \varepsilon \sup _{\mathbf{x}}|\nabla \mathbf{X}|^{2},
$$

where $C$ depends only on the curvature of $\Sigma$, and (3.23) is therefore true.

Now in order to compute the value of $\mathcal{F}_{2}\left(K_{\varepsilon}\right)$ we decompose the domain $K \triangle K_{\varepsilon}$ in two parts

$$
K \triangle K_{\varepsilon}=\left(K \triangle K_{\varepsilon} \cap\left\{\left\langle\mathbf{X}\left(\pi^{\Sigma}(\mathbf{x})\right), \mathbf{n}\right\rangle \geq 0\right\}\right) \cup\left(K \triangle K_{\varepsilon} \cap\left\{\left\langle\mathbf{X}\left(\pi^{\Sigma}(\mathbf{x})\right), \mathbf{n}\right\rangle<0\right\}\right) .
$$

Let us consider the integral on $K \triangle K_{\varepsilon} \cap\left\{\left\langle\mathbf{X}\left(\pi^{\Sigma}(\mathbf{x})\right), \mathbf{n}\right\rangle \geq 0\right\}$. Recall that $\partial K \cap \operatorname{supp}(\mathbf{X})$ is supposed to be regular, at least $C^{2}$. Let $\mathbf{f}:\left[\ell_{1}, \ell_{2}\right] \rightarrow \partial K$ be the parametrization by arc length in correspondence of one connected component of $\left(\langle\mathbf{X}, \mathbf{n}\rangle \circ \pi^{\Sigma}\right)^{-1}\left(\mathbb{R}^{+}\right) \cap\left(K \triangle K_{\varepsilon}\right)$ (denoted by $\left.A_{\varepsilon}\right)$ and consider the mapping

$$
\varphi:(t, y) \mapsto \mathbf{f}(t)+y \mathbf{n}(t)
$$

where $\mathbf{n}(t)=-\frac{\mathbf{f}_{2}(t)}{\left|\mathbf{f}_{2}(t)\right|}$ is the unit outward normal vector at point $\mathbf{f}(t)$. The mapping $\varphi$ is a diffeomorphism from $\varphi^{-1}\left(A_{\varepsilon}\right)$ to $A_{\varepsilon}$. Since $\mathbf{f}_{1}(t)$ is the unit tangent vector at point $\mathbf{f}(t)$ and $\mathbf{n}(t)^{\prime}=-H(t) \mathbf{f}_{1}(t)$, where $H(t)$ is the curvature of the curve $\mathbf{f}(t)$, we have that $D \varphi$ is the matrix with columns $(1-\gamma(t) y) \mathbf{f}_{1}(t)$ and $\mathbf{n}(t)$. Therefore, $|J \Phi|(t, y)=|1-H(t) y|=1-H(t) y$ for $\varepsilon$ small enough. Thus using (3.23) and a change of variable we get

$$
\begin{aligned}
\int_{A_{\varepsilon}} \operatorname{dist}\left(\mathbf{x}, \partial K_{\varepsilon}\right) \mathrm{d} \mathbf{x} & =\int_{A_{\varepsilon}}\left(\varepsilon\left|\mathbf{X}\left(\pi^{\Sigma}(\mathbf{x})\right)\right|-\operatorname{dist}(\mathbf{x}, \partial K)+o(\varepsilon)\right) \mathrm{d} \mathbf{x} \\
& =\int_{\ell_{1}}^{\ell_{2}} \int_{0}^{\varepsilon|\mathbf{X}(\mathbf{f}(t))|}(\varepsilon|\mathbf{X}(\mathbf{f}(t))|-y)(1-H(t) y) \mathrm{d} y \mathrm{~d} t \\
& =\frac{1}{2} \varepsilon^{2} \int_{\ell_{1}}^{\ell_{2}}|\mathbf{X}(\mathbf{f}(t))|^{2} \mathrm{~d} t+o\left(\varepsilon^{2}\right) .
\end{aligned}
$$


Arguing on each connected component, and using the same argument for $\left(\langle\mathbf{X}, \mathbf{n}\rangle \circ \pi^{\Sigma}\right)^{-1}\left(\mathbb{R}^{-}\right) \cap\left(K \triangle K_{\varepsilon}\right)$ we obtain that

$$
\lim _{\varepsilon \rightarrow 0} \frac{1}{\varepsilon^{2}} \mathcal{F}_{2}\left(K_{\varepsilon}\right)=\frac{1}{2} \int_{\partial K} \operatorname{sign}\langle\mathbf{X}(\mathbf{x}), \mathbf{n}\rangle|\mathbf{X}(\mathbf{x})|^{2} \mathrm{~d} \mathcal{H}^{1}(\mathbf{x}),
$$

which proves, since $\left.\mathcal{F}_{2}\left(K_{\varepsilon}\right)\right|_{\varepsilon=0}=\left.\frac{\mathrm{d}}{\mathrm{d} \varepsilon} \mathcal{F}_{2}\left(K_{\varepsilon}\right)\right|_{\varepsilon=0}=0$, that

$$
\left.\frac{\mathrm{d}^{2}}{\mathrm{~d} \varepsilon^{2}} \mathcal{F}_{2}\left(K_{\varepsilon}\right)\right|_{\varepsilon=0}=\int_{\partial K} \operatorname{sign}\langle\mathbf{X}(\mathbf{x}), \mathbf{n}\rangle|\mathbf{X}(\mathbf{x})|^{2} \mathrm{~d} \mathcal{H}^{1}(\mathbf{x}) .
$$

The proof is concluded.

In order to obtain second order necessary conditions for minimality, of course we ask the second derivative of $\mathcal{F}(\cdot)+\lambda_{1} \operatorname{Vol}(\cdot)+\lambda_{2} \operatorname{Per}(\cdot)$ to be nonnegative. Again, the second derivatives of $\operatorname{Per}\left(K_{\varepsilon}\right)$ and $\operatorname{Vol}\left(K_{\varepsilon}\right)$ might be deduced from the general theory of [13], Chapter 5. For the derivatives of the perimeter, see also [20]. Here we are restricting to the particular case of normal vector fields.

Corollary 3.9 (second order necessary conditions). In the problem with perimeter penalization, for a $C^{2}$ set $K \subseteq \Omega$ satisfying (3.20), a second order minimality condition is

$$
\mathcal{G}_{n}(K)-\int_{\partial K} \operatorname{sign}\langle\mathbf{X}, \mathbf{n}\rangle|\mathbf{X}|^{2} \mathrm{~d} \mathcal{H}^{1}+\lambda_{2} \int_{\partial K}\left|\partial_{\tau} \mathbf{X}\right|^{2} \mathrm{~d} \mathcal{H}^{1} \geq 0
$$

for any normal vector field $\mathbf{X}$. In the problem with volume penalization, for a strictly convex $C^{2}$ set $K \subseteq \Omega$ satisfying (3.21), it is

$$
\mathcal{G}_{n}(K)-\int_{\partial K} \operatorname{sign}\langle\mathbf{X}, \mathbf{n}\rangle|\mathbf{X}|^{2} \mathrm{~d} \mathcal{H}^{1}+\lambda_{1} \int_{\partial K} H|\mathbf{X}|^{2} \mathrm{~d} \mathcal{H}^{1} \geq 0
$$

for any normal vector field $\mathbf{X}$.

\section{ExClusion OF STRICTLY CONVEX $C^{2}$-POINTS FOR THE VOlume PENALized PROBLEM}

Definition 4.1. Let $K \subseteq \mathbb{R}^{2}$ be convex. We will say that $\mathbf{x}_{0} \in \partial K$ is a strictly convex $C^{2}$-point if $\mathbf{x}_{0} \in \Omega \cap \partial K$, $\partial K$ is $C^{2}$-regular in a neighborhood of $\mathbf{x}_{0}$ and the curvature of $K$ at $\mathbf{x}_{0}$ is positive.

Theorem 4.2. Assume that $K$ is a minimizer for problem (1.2) with $\lambda_{1}>0$ and $\lambda_{2}=0$. Then $\partial K$ has no strictly convex $C^{2}$-points.

Proof. Assume by contradiction that such a point $\mathbf{x}_{0} \in K$ exists. In a small enough neighborhood $V\left(\mathbf{x}_{0}\right)$ of $\mathbf{x}_{0}$ we know that $\partial K$ is a graph. Let us consider a system of coordinates where $\mathbf{x}_{0}$ is the origin, the $x$ axis and the $y$ axes are respectively tangent and normal to $\partial K$ at $\mathbf{x}_{0}$ and

$$
\partial K \cap\left(\left\{-\alpha_{0} \leq x \leq \alpha_{0}\right\} \times\{y \leq 0\}\right)=\left\{(x, f(x)), x \in\left[-\alpha_{0}, \alpha_{0}\right]\right\} \subseteq V\left(\mathbf{x}_{0}\right)
$$

for some $C^{2}$ function $f:[-1,1] \rightarrow \mathbb{R}^{-}$and a suitable $\alpha_{0}>0$. We have $f^{\prime}(0)=0$ and we also can assume without loss of generality (taking a small enough $\alpha_{0}$ ) that

$$
\sup _{x \in\left[-\alpha_{0}, \alpha_{0}\right]}\left(|| f^{\prime \prime}(x)|-| f^{\prime \prime}(0)||\right) \leq \delta,
$$

for a given small $\delta$, so that $\partial K \cap V\left(\mathbf{x}_{0}\right)$ is almost of constant curvature. Therefore $f(x)$ is very close to the graph of $x \mapsto f^{\prime \prime}(0) \frac{x^{2}}{2}$ on the interval $\left[-\alpha_{0}, \alpha_{0}\right]$, and $K$ lies below this graph. We deduce that, if $0 \leq \alpha \leq \alpha_{0}$,

$$
\sup _{t \in[-\alpha, \alpha]}|f(t)| \leq \frac{1}{2} \alpha^{2}\left(\left|f^{\prime \prime}(0)\right|+\delta\right):=b .
$$


Let us now consider a smooth vector field $\mathbf{X}: \mathbb{R}^{2} \rightarrow \mathbb{R}^{2}$ compactly supported in $[-\alpha, \alpha] \times[-b, b]$, normal to $\partial K$. Then we consider the set $K_{\varepsilon}:=(\operatorname{Id}+\varepsilon \mathbf{X})(K)$, which remain convex for $\varepsilon$ small enough because $f^{\prime \prime}(0) \neq 0$ by assumption. In this situation, (3.18) directly says that

$$
\left.\frac{\mathrm{d}}{\mathrm{d} \varepsilon} \int_{\Omega} \operatorname{dist}\left(\mathbf{x}, K_{\varepsilon}\right) \mathrm{d} \mathbf{x}\right|_{\varepsilon=0}=-\int_{\Omega \backslash K}\left\langle\mathbf{X}\left(\pi^{\partial K}(\mathbf{x})\right), \mathbf{n}\left(\pi^{\partial K}(\mathbf{x})\right)\right\rangle \mathrm{d} \mathbf{x}
$$

$\mathbf{n}$ being the outward unit normal, and (3.22) yields,

$$
\begin{aligned}
\left.\frac{\mathrm{d}^{2}}{\mathrm{~d} \varepsilon^{2}} \int_{\Omega} \operatorname{dist}\left(\mathbf{x}, K_{\varepsilon}\right) \mathrm{d} \mathbf{x}\right|_{\varepsilon=0}= & -\int_{\Omega \backslash K} \frac{\operatorname{dist}(\mathbf{x}, K)\left|\partial_{\tau} \mathbf{X}\left(\pi^{\partial K}(\mathbf{x})\right)\right|^{2}}{2\left(1+\operatorname{dist}(\mathbf{x}, K) \mid f^{\prime \prime}\left(\pi^{\partial K}(\mathbf{x})_{1}\right)\right) \mid} \mathrm{d} \mathbf{x} \\
& -\int_{\partial K} \operatorname{sign}\langle\mathbf{X}(\mathbf{x}), \mathbf{n}(\mathbf{x})\rangle|\mathbf{X}(\mathbf{x})|^{2} \mathrm{~d} \mathcal{H}^{1}(\mathbf{x}),
\end{aligned}
$$

where $\pi^{\partial K}(\mathbf{x})_{1}$ is the first coordinate of the projected point $\pi^{\partial K}(\mathbf{x})$ and $\partial_{\tau}$ denotes the tangential derivative. The derivatives of $\operatorname{Vol}\left(K_{\varepsilon}\right)$ are well known (see [13], Chap. 5): we have

$$
\left.\frac{\mathrm{d}}{\mathrm{d} \varepsilon} \operatorname{Vol}\left(K_{\varepsilon}\right)\right|_{\varepsilon=0}=\int_{K} \operatorname{div} \mathbf{X} \mathrm{d} \mathbf{x}=\int_{\partial K}\langle\mathbf{X}, \mathbf{n}\rangle \mathrm{d} \mathcal{H}^{1},\left.\quad \frac{\mathrm{d}^{2}}{\mathrm{~d} \varepsilon^{2}} \operatorname{Vol}\left(K_{\varepsilon}\right)\right|_{\varepsilon=0}=\int_{\partial K} H|\mathbf{X}|^{2} \mathrm{~d} \mathcal{H}^{1},
$$

as already seen in (3.21) and (3.26), where $H$ is the mean curvature. Therefore, the first order condition gives

$$
\lambda_{1} \int_{\partial K}\langle\mathbf{X}(\mathbf{x}), \mathbf{n}(\mathbf{x})\rangle \mathrm{d} \mathcal{H}^{1}(\mathbf{x})=\int_{\Omega \backslash K}\left\langle\mathbf{X}\left(\pi^{\partial K}(\mathbf{x})\right), \mathbf{n}\left(\pi^{\partial K}(\mathbf{x})\right)\right\rangle \mathrm{d} \mathbf{x}
$$

and the second order condition implies

$$
\begin{aligned}
\lambda_{1} \int_{\partial K} H(\mathbf{x})|\mathbf{X}(\mathbf{x})|^{2} \mathrm{~d} \mathcal{H}^{1}(\mathbf{x}) & -\int_{\Omega \backslash K} \frac{\operatorname{dist}(\mathbf{x}, K)\left|\partial_{\tau} \mathbf{X}\left(\pi^{\partial K}(\mathbf{x})\right)\right|^{2}}{\left(1+\operatorname{dist}(\mathbf{x}, K)\left|f^{\prime \prime}\left(\pi^{\partial K}(\mathbf{x})_{1}\right)\right|\right)} \mathrm{d} \mathbf{x} \\
& -\int_{\partial K} \operatorname{sign}\langle\mathbf{X}(\mathbf{x}), \mathbf{n}(\mathbf{x})\rangle|\mathbf{X}(\mathbf{x})|^{2} \mathrm{~d} \mathcal{H}^{1}(\mathbf{x}) \geq 0 .
\end{aligned}
$$

To prove the Theorem we will show that (4.2) and (4.3) cannot be verified for every choices of $\mathbf{X}$. Let $\alpha<\alpha_{0}$. Let $\Gamma_{\alpha}:=\partial K \cap[-\alpha / 2, \alpha / 2] \times \mathbb{R}$ and take a particular family of smooth vector fields $\mathbf{X}_{\alpha}$ satisfying

$$
\begin{array}{ll}
\text { (i) } & \inf _{\mathbf{x} \in \Gamma_{\alpha}}\left|\partial_{\tau} \mathbf{X}_{\alpha}(\mathbf{x})\right| \geq \frac{C_{1}}{\alpha}, \\
\text { (ii) } & \operatorname{supp}\left(\mathbf{X}_{\alpha}\right) \subseteq[-\alpha, \alpha] \times[-b, b], \\
\text { (iii) } & \sup _{\mathbf{x} \in \mathbb{R}^{2}}\left|\mathbf{X}_{\alpha}(\mathbf{x})\right| \leq C_{2},
\end{array}
$$

for some positive constants $C_{1}$ and $C_{2}$. Here $\alpha>0$ is a small number, and since $b$ is defined by (4.1), we see that the requests (i), (ii) and (iii) are compatible.

Next, recalling that $\left|f^{\prime \prime}(x)\right| \leq\left|f^{\prime \prime}(0)\right|+\delta$, by (i) and (iii) we deduce that

$$
\begin{aligned}
& \lambda_{1} \int_{\partial K} H\left|\mathbf{X}_{\alpha}\right|^{2} \mathrm{~d} \mathcal{H}^{1}-\int_{\partial K} \operatorname{sign}\left\langle\mathbf{X}_{\alpha}, \mathbf{n}\right\rangle|\mathbf{X}|^{2} \mathrm{~d} \mathcal{H}^{1}-\int_{\Omega \backslash K} \frac{\operatorname{dist}(\mathbf{x}, K)\left|\partial_{\tau} \mathbf{X}_{\alpha}\left(\pi^{\partial K}(\mathbf{x})\right)\right|^{2}}{\left(1+\operatorname{dist}(\mathbf{x}, K)\left|f^{\prime \prime}\left(\pi^{\partial K}(\mathbf{x})_{1}\right)\right|\right)} \mathrm{d} \mathbf{x} \\
& \leq \lambda_{1} C_{2}^{2} C_{3} \alpha+C_{3} C_{2}^{2} \alpha-\frac{C_{1}^{2}}{\alpha^{2}} \int_{\Omega \cap(\{-\alpha / 2 \leq x \leq \alpha / 2\} \times\{y \geq f(x)\})} \frac{\operatorname{dist}(\mathbf{x}, K)}{\left(1+\operatorname{dist}(\mathbf{x}, K)\left(\left|f^{\prime \prime}(0)\right|\right)+\delta\right)} \mathrm{d} \mathbf{x},
\end{aligned}
$$


where $C_{3}$ is a new positive constant, which depends only on $K$ and $\delta$. We claim that this last term is negative provided $\alpha$ is small enough. Indeed we have

$$
\int_{\Omega \cap(\{-\alpha / 2 \leq x \leq \alpha / 2\} \times\{y \geq f(x)\})} \frac{\operatorname{dist}(\mathbf{x}, K)}{\left(1+\operatorname{dist}(\mathbf{x}, K)\left(\left|f^{\prime \prime}(0)\right|\right)+\delta\right)} \mathrm{d} \mathbf{x} \geq C_{4} \alpha,
$$

where $C_{4}$ is another suitable positive constant, depending only on $\Omega, K$ and the mean value of the integrand (which itself depends only on $K$ ). Thus the last term in (4.5) is smaller than $C_{2}^{2} C_{3} \lambda_{1} \alpha+C_{3} C_{2}^{2} \alpha-\frac{C_{1}^{2} C_{4}}{\alpha}$ which is clearly negative for $\alpha$ small enough, and the Theorem is proved.

Remark 4.3 (the case of perimeter penalization). In this case, the first and second order derivatives of the perimeter (see [13]), appearing in (3.20) and (3.25), are

$$
\left.\frac{\mathrm{d}}{\mathrm{d} \varepsilon} \operatorname{Per}\left(K_{\varepsilon}\right)\right|_{\varepsilon=0}=\int_{\partial K} H\langle\mathbf{X}, \mathbf{n}\rangle \mathrm{d} \mathcal{H}^{1}, \quad \text { and }\left.\quad \frac{\mathrm{d}^{2}}{\mathrm{~d} \varepsilon^{2}} \operatorname{Per}\left(K_{\varepsilon}\right)\right|_{\varepsilon=0}=\int_{\partial K}\left|\partial_{\tau} \mathbf{X}\right|^{2} \mathrm{~d} \mathcal{H}^{1} .
$$

As a consequence the analogue of (4.5) would contain two terms of same order which prevents us to exclude strictly convex points with the same method.

\section{THE CASE OF POLYGONS}

In the previous section we saw that minimizers cannot admit strictly convex points at their boundary. Therefore, it is natural to consider the question whether a polygon could be a minimizer or not. We begin with the following proposition about the problem with perimeter constraint only, coming from a first order argument.

Lemma 5.1. A polygon compactly contained in $\Omega$ is never a minimizer for the problem (1.2) if $\lambda_{1}=0$.

Proof. We denote by $\mathbf{e}_{1}$ and $\mathbf{e}_{2}$ the two unit vectors associated with the axis of $\mathbb{R}^{2}$. A generic point $\mathbf{x} \in \mathbb{R}^{2}$ will have coordinates $\mathbf{x}=(x, y)$. Let $K$ be a polygon and $S$ be one of its edges. By scale and rotation invariance we can assume without loss of generality that $S$ is the interval $[0,1] \times\{0\}$ and $K$ is below the first axis, i.e. $K \subseteq\{y \leq 0\}$. Notice that from Remark 2.3 we are allowed to take any competitor for $K$, not necessarily convex. Let $\zeta \in C_{0}^{\infty}([0,1], \mathbb{R})$ be a smooth function, compactly supported in $[0,1]$.

Then we define the vector field $\mathbf{X}$ normal to $S$ by setting first

$$
\tilde{\mathbf{X}}(\mathbf{x})=\zeta(x) \mathbf{e}_{2},
$$

and then $\mathbf{X}:=\psi \tilde{\mathbf{X}}$ where $\psi \in C_{0}^{\infty}\left(\mathbb{R}^{2}, \mathbb{R}\right)$ is a cut-off function which is equal to 1 in a neighborhood of $S$. We denote as usual $\Phi_{\varepsilon}:=\mathrm{Id}+\varepsilon \mathbf{X}$ and $K_{\varepsilon}:=\Phi_{\varepsilon}(K)$. Notice that the only part of $\partial K$ that is moved by $\Phi_{\varepsilon}$ is the edge $S$. All the rest of the polygon remains the same.

We have that

$$
\begin{aligned}
\operatorname{Per}\left(K_{\varepsilon}\right) & =\operatorname{Per}(K)+\left(\int_{0}^{1} \sqrt{1+\left|\varepsilon \zeta^{\prime}(t)\right|^{2}} \mathrm{~d} t-1\right) \\
& =\operatorname{Per}(K)+\varepsilon^{2} \int_{0}^{1} \frac{\left|\zeta^{\prime}(t)\right|^{2}}{2} \mathrm{~d} t+o\left(\varepsilon^{2}\right) .
\end{aligned}
$$

Therefore,

$$
\left.\frac{\mathrm{d}}{\mathrm{d} \varepsilon} \operatorname{Per}\left(K_{\varepsilon}\right)\right|_{\varepsilon=0}=0
$$

Next let us see what happens with the average distance. Let us define

$$
f_{\Omega}(t):=\sup \{y ;(t, y) \in \Omega\} .
$$


Then by (3.2) we infer that

$$
\left.\frac{\mathrm{d}}{\mathrm{d} \varepsilon}\left(\int_{\Omega} \operatorname{dist}\left(\mathbf{x}, K_{\varepsilon}\right) \mathrm{d} \mathbf{x}\right)\right|_{\varepsilon=0}=-\int_{0}^{1} f_{\Omega}(t) \zeta(t) \mathrm{d} t .
$$

Joining together the previous computations, if the polygon was a minimizer for the problem (1.2) we would have at first order that

$$
0=-\int_{0}^{1} f_{\Omega}(t) \zeta(t) \mathrm{d} t
$$

We see that the polygon cannot satisfy the first order condition in the case of perimeter penalization.

Remark 5.2. Actually the proof of Proposition 5.1 excludes any "flat part" on the boundary of a minimizer $K$ for the functional $\mathcal{F}(K)+\lambda_{2} \operatorname{Per}(K)$, provided this flat part does not touch the boundary of $\Omega$.

Remark 5.3. About the problem with volume constraint (i.e. $\lambda_{1}>0$ and $\lambda_{2}=0$ ), in the situation of Proposition 5.1 it is clear that there is

$$
\left.\frac{\mathrm{d}}{\mathrm{d} \varepsilon} \operatorname{Vol}\left(K_{\varepsilon}\right)\right|_{\varepsilon=0}=\varepsilon \int_{0}^{1} \zeta(t) \mathrm{d} t .
$$

But this time, since we are only allowed to take unilateral variations (since we have to keep convexity for a non strictly convex set), the first order condition of Proposition 5.1 would be only an inequality:

$$
0 \leq \lambda_{1} \int_{0}^{1} \zeta(t) \mathrm{d} t-\int_{0}^{1} f_{\Omega}(t) \zeta(t) \mathrm{d} t
$$

When the inequality is strict, no second order condition is possible for this particular variation. On the other hand the following proposition provides an example of a polygon and a variation which satisfy both first and second order condition for the problem with volume constraint.

Proposition 5.4 (first and second order variation of squares for side translation). Consider the problem with volume penalization (that is, $\lambda_{2}=0$ ). There exists $\lambda_{1}>0$ such that the square $K:=[0,1] \times[-1,0] \subseteq \Omega$ satisfies both first and second order conditions for a particular deformation $K_{\varepsilon}$ of $K$.

Proof. Let $\varepsilon \in \mathbb{R}$. Let $K:=[0,1] \times[-1,0] \subseteq \Omega$ for some domain $\Omega$ and consider the variation $K_{\varepsilon}:=[0,1] \times$ $[-1,-\varepsilon]$. This corresponds to a variation given by the diffeomorphism $\operatorname{Id}+\varepsilon \mathbf{X}$, where the vector field $\mathbf{X}$ is smooth, compactly supported in a neighborhood of the edge $[0,1] \times\{0\}$ and directed as $\mathbf{e}_{2}$. On the edge $[0,1] \times\{0\}$ it is given by $-\mathbf{e}_{2}$. Now we want to compute the first and second order derivative of $\mathcal{F}\left(K_{\varepsilon}\right)+\lambda_{1} \operatorname{Vol}\left(K_{\varepsilon}\right)$ with respect to $\varepsilon$. For the volume we have

$$
\left.\frac{\mathrm{d}}{\mathrm{d} \varepsilon} \operatorname{Vol}\left(K_{\varepsilon}\right)\right|_{\varepsilon=0}=-1 \quad \text { and }\left.\quad \frac{\mathrm{d}^{2}}{\mathrm{~d} \varepsilon^{2}} \operatorname{Vol}\left(K_{\varepsilon}\right)\right|_{\varepsilon=0}=0
$$

For the average distance, from (3.2) we deduce that

$$
\left.\frac{\mathrm{d}}{\mathrm{d} \varepsilon} \mathcal{F}\left(K_{\varepsilon}\right)\right|_{\varepsilon=0}=C_{1}(\Omega)+\int_{\left(\pi^{K}\right)^{-1}((0,0) \cup(1,0))} \cos (\alpha(\mathbf{x})) \mathrm{d} \mathbf{x},
$$

where $C_{1}(\Omega)=\operatorname{Vol}(\Omega \cap[0,1] \times\{y \geq 0\})$ and $\alpha(\mathbf{x})$ is the angle between $\mathbf{x}-\pi^{K}(\mathbf{x})$ and the second unit vector $\mathbf{e}_{2}$. In particular the first order condition is

$$
\lambda_{1}-C_{1}(\Omega)=\int_{\left(\pi^{K}\right)^{-1}((0,0) \cup(1,0))} \cos (\alpha(\mathbf{x})) \mathrm{d} \mathbf{x} .
$$


Now assume that $K$ is stationary for this deformation, i.e. that $\lambda_{1}$ verifies equality (5.3). We also check with some computations that $K$ satisfies the second order condition. We will not make use of the general formulas of Section 3, since we do not have a smooth enough set $K$. Let us divide $\Omega \backslash K_{\varepsilon}$ in four parts $A_{\varepsilon}, B_{\varepsilon}, C_{\varepsilon}$ and $\Omega \backslash\left(A_{\varepsilon} \cup B_{\varepsilon} \cup C_{\varepsilon}\right)$ where

$$
\begin{aligned}
& A_{\varepsilon}:=\{(x, y) \in \Omega: 0 \leq x \leq 1, y \geq \min \{0,-\varepsilon\}\} \\
& B_{\varepsilon}:=\{(x, y) \in \Omega: x \notin[0,1], y \geq \max \{0,-\varepsilon\}\} \\
& C_{\varepsilon}:=\{(x, y) \in \Omega: x \notin[0,1], \min \{0,-\varepsilon\}<y<\max \{0,-\varepsilon\}\}
\end{aligned}
$$

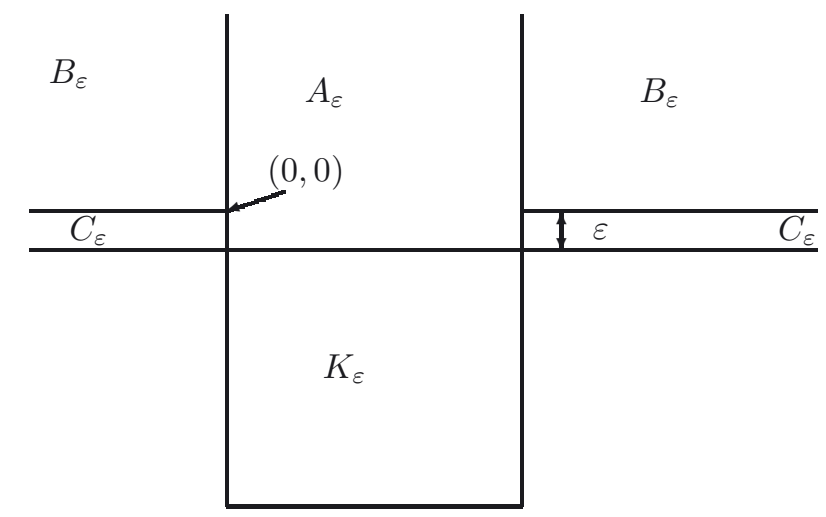

By this way all the points in $\Omega \backslash\left(K_{\varepsilon} \cup A_{\varepsilon} \cup B_{\varepsilon} \cup C_{\varepsilon}\right)$ are still projected on the same point belonging to $K$ and we only need to compute the variations of the distance functional on $A_{\varepsilon} \cup B_{\varepsilon} \cup C_{\varepsilon}$. In the following, we will compute the right derivative, i.e. we will consider the case $\varepsilon>0$. The computation of the left second derivative is substantially analogous, we will omit the details, and of course its value is the same we are going to obtain for the second order right derivative.

We begin with the contribution of points in $A_{\varepsilon}$. They are all projected on $K_{\varepsilon} \cap\{y=-\varepsilon\}$ which leads to

$$
\begin{aligned}
\int_{A_{\varepsilon}} \operatorname{dist}\left(\mathbf{x}, K_{\varepsilon}\right) & =\int_{A_{\varepsilon} \cap\{y \geq 0\}} \operatorname{dist}\left(\mathbf{x}, K_{\varepsilon}\right)+\int_{A_{\varepsilon} \cap\{-\varepsilon \leq y \leq 0\}} \operatorname{dist}\left(\mathbf{x}, K_{\varepsilon}\right) \\
& =\int_{A_{\varepsilon} \cap\{y \geq 0\}}(\operatorname{dist}(\mathbf{x}, K)+\varepsilon)+\int_{0}^{1} \int_{-\varepsilon}^{0}(y+\varepsilon) \mathrm{d} y \mathrm{~d} x \\
& =\int_{A_{\varepsilon} \cap\{y \geq 0\}} \operatorname{dist}(\mathbf{x}, K)+\varepsilon C_{1}(\Omega)+\frac{\varepsilon^{2}}{2},
\end{aligned}
$$

and therefore

$$
\left.\frac{\mathrm{d}^{2}}{\mathrm{~d} \varepsilon^{2}} \int_{A_{\varepsilon}} \operatorname{dist}\left(\mathbf{x}, K_{\varepsilon}\right) \mathrm{d} \mathbf{x}\right|_{\varepsilon=0}=1 .
$$

Next we claim that the integral on $C_{\varepsilon}$ is of order higher than $\varepsilon^{2}$. Indeed, for each point in $C_{\varepsilon}$, using Pythagoras and the equality $\sqrt{1+a^{2}}=1+\frac{a^{2}}{2}+o\left(a^{2}\right)$ we see that

$$
\operatorname{dist}\left(\mathbf{x}, K_{\varepsilon}\right)=\sqrt{x^{2}+y^{2}+2 \varepsilon y+\varepsilon^{2}}=|\mathbf{x}|+\frac{2 \varepsilon y+\varepsilon^{2}}{2|\mathbf{x}|}+\frac{o\left(\varepsilon^{2}\right)}{|\mathbf{x}|}
$$


as $\varepsilon \downarrow 0$, since $|y| \leq \varepsilon$. By this fact, together with the integrability of $\frac{1}{|\mathbf{x}|}$ in two dimensions, we see that integrating over the region $C_{\varepsilon}$, whose volume is of order $\varepsilon$, there is

$$
\int_{C_{\varepsilon}}|\mathbf{x}| \mathrm{d} \mathbf{x}=Q_{\Omega} \varepsilon \quad \text { and } \quad \int_{C_{\varepsilon}} \frac{2 \varepsilon y+\varepsilon^{2}+o\left(\varepsilon^{2}\right)}{2|\mathbf{x}|} \mathrm{d} \mathbf{x}=o\left(\varepsilon^{2}\right),
$$

where $Q_{\Omega}$ is a suitable constant depending only on $\Omega$. We see that there is no second order term:

$$
\int_{C_{\varepsilon}} \operatorname{dist}\left(\mathbf{x}, K_{\varepsilon}\right)=Q_{\Omega} \varepsilon+o\left(\varepsilon^{2}\right) .
$$

It remains to count the contribution on the domain $B_{\varepsilon}$. Notice that $B_{\varepsilon}$ does not depend on $\varepsilon$ for $\varepsilon>0$, and is equal to $B_{0}:=\{(x, y) \in \Omega: x \notin[0,1], y \geq 0\}$. Let us consider first the domain $B_{\varepsilon} \cap\{x<0\}$, the domain $B_{\varepsilon} \cap\{x>0\}$ can be treated the same way. If $\mathbf{x} \in B_{\varepsilon} \cap\{x<0\}$, then $\operatorname{dist}\left(\mathbf{x}, K_{\varepsilon}\right)=\operatorname{dist}(\mathbf{x},(0,-\varepsilon))$ and therefore

$$
\operatorname{dist}\left(\mathbf{x}, K_{\varepsilon}\right)^{2}=\operatorname{dist}(\mathbf{x}, K)^{2}+\varepsilon^{2}+2 \varepsilon \cos \alpha(\mathbf{x}) \operatorname{dist}(\mathbf{x}, K) .
$$

Next, making use of (3.9) a simple computation yields

$$
\begin{aligned}
\int_{B_{\varepsilon} \cap\{x<0\}} \operatorname{dist}\left(\mathbf{x}, K_{\varepsilon}\right) \mathrm{d} \mathbf{x}= & \int_{B_{\varepsilon} \cap\{x<0\}} \operatorname{dist}(\mathbf{x}, K) \mathrm{d} \mathbf{x}+\varepsilon \int_{B_{\varepsilon} \cap\{x<0\}} \cos (\alpha(\mathbf{x})) \mathrm{d} \mathbf{x} \\
& +\frac{\varepsilon^{2}}{2} \int_{B_{\varepsilon} \cap\{x<0\}} \frac{\sin ^{2} \alpha(\mathbf{x})}{\operatorname{dist}(\mathbf{x}, K)} \mathrm{d} \mathbf{x}+o\left(\varepsilon^{2}\right),
\end{aligned}
$$

and the same holds for $B_{\varepsilon} \cap\{x>0\}$, from which we obtain that

$$
\left.\frac{\mathrm{d}^{2}}{\mathrm{~d} \varepsilon^{2}} \int_{B_{\varepsilon}} \operatorname{dist}\left(\mathbf{x}, K_{\varepsilon}\right) \mathrm{d} \mathbf{x}\right|_{\varepsilon=0}=\int_{B_{0}} \frac{\sin ^{2} \alpha(\mathbf{x})}{\operatorname{dist}(\mathbf{x}, K)} \mathrm{d} \mathbf{x} .
$$

Again, the integral in the right hand side of $(5.8)$ is finite because $\frac{1}{\operatorname{dist}(\mathbf{x}, K)}$ behaves like $\frac{1}{|\mathbf{x}|}$, which is integrable at the origin in dimension 2. Finally putting together (5.5), (5.6) and (5.8) we have that

$$
\left.\frac{\mathrm{d}^{2}}{\mathrm{~d} \varepsilon^{2}} \int_{\Omega} \operatorname{dist}\left(\mathbf{x}, K_{\varepsilon}\right) \mathrm{d} \mathbf{x}\right|_{\varepsilon=0}=1+\int_{B_{0}} \frac{\sin ^{2} \alpha(\mathbf{x})}{\operatorname{dist}(\mathbf{x}, K)} \mathrm{d} \mathbf{x}>0
$$

which proves that the second order minimality condition is fulfilled.

Remark 5.5. The above proposition actually proves that, in comparison with rectangles, only the square is a local minimizer in its $\varepsilon$-neighborhood. Indeed, if the edges have different length, a rectangle could not satisfy the first order condition (5.3) in its $\varepsilon$-neighborhood for translation of each edge. We could probably do the same for any regular polygon but the case of the rectangle is much simpler because of the orthogonality at the corners. Of course the previous proposition does not prove that regular polygons are minimizers, but that they are local minimizers for the considered class of variations.

\section{Approximation by Gamma-Convergence}

In this last section we approximate the average distance functional from a convex set by a certain family of elliptic functionals, in view of numerical computations, as for e.g. Ambrosio and Tortorelli [2] did for the Mumford-Shah functional. For this purpose we will use the result of Buttazzo and Santambrogio [5], which says that the average distance functional can be obtained as the limit when $p \rightarrow+\infty$ of the $p$-compliance functional. We will also use the seminal work of Modica and Mortola [17] for the perimeter term. 
In the sequel $\Omega$ will denote a bounded domain of $\mathbb{R}^{2}$. For any $p>1, \varphi \in L^{\infty}(\Omega)$ and $\lambda>0$, we denote by $u_{\varphi, \lambda, p}$ the unique solution $u \in W^{1, p}(\Omega)$ of the problem

$$
-\Delta_{p} u+\lambda \varphi u=1
$$

The solution $u_{\varphi, \lambda, p}$ can be obtained for instance by minimizing the energy

$$
E_{\varphi, \lambda, p}(u):=\frac{1}{p} \int_{\Omega}|\nabla u|^{p} \mathrm{~d} \mathbf{x}-\left(\int_{\Omega} u-\lambda \varphi u^{2} \mathrm{~d} \mathbf{x}\right)
$$

among all functions $u \in W^{1, p}(\Omega)$.

As a first step to approximate our minimization problem we begin with the following $\Gamma$-convergence result which is a little inspired from a lemma given without proof in [5], Lemma 7.

Proposition 6.1. Let $\varphi_{\varepsilon}: \Omega \rightarrow \mathbb{R}^{+}$be some nonnegative measurable bounded functions converging to some $\varphi \in L^{\infty}(\Omega)$ when $\varepsilon \rightarrow 0$, and assume also that $p_{\varepsilon} \rightarrow+\infty$. Then the family of functionals $E_{\varphi_{\varepsilon}, \lambda, p_{\varepsilon}} \Gamma$-converges in $L^{2}$ to the functional

$$
E_{\varphi, \lambda}(u):=\left\{\begin{array}{c}
-\int_{\Omega} u+\lambda \int_{\Omega} \varphi u^{2} \quad \text { if } u \in W^{1, \infty} \text { and }\|\nabla u\|_{L^{\infty}} \leq 1 \\
+\infty \\
\text { otherwise. }
\end{array}\right.
$$

Proof. The limsup inequality is fairly simple. Let $u \in W^{1, \infty}$ with $\|\nabla u\|_{\infty} \leq 1$ be given. We have to find a recovery sequence $u_{\varepsilon}$ converging to $u$ in $L^{2}$ and such that

$$
\limsup _{\varepsilon \rightarrow 0} E_{\varphi_{\varepsilon}, \lambda, p_{\varepsilon}}\left(u_{\varepsilon}\right) \leq E_{\varphi, \lambda}(u) .
$$

We simply take the identically constant sequence $u_{\varepsilon}=u$. Since $|\nabla u| \leq 1$ we easily obtain that

$$
\frac{1}{p_{\varepsilon}} \int_{\Omega}|\nabla u|^{p_{\varepsilon}} \rightarrow 0
$$

Therefore, since

$$
E_{\varphi_{\varepsilon}, \lambda, p_{\varepsilon}}\left(u_{\varepsilon}\right)=\frac{1}{p_{\varepsilon}} \int_{\Omega}|\nabla u|^{p_{\varepsilon}}-\int_{\Omega} u+\lambda \int_{\Omega} \varphi_{\varepsilon} u^{2}
$$

we conclude taking the limsup, actually proving (6.2) with an equality.

Now to prove the liminf inequality, we consider a sequence $u_{\varepsilon} \rightarrow u$ in $L^{2}$. We have to prove that

$$
E_{\varphi, \lambda}(u) \leq \liminf _{\varepsilon \rightarrow 0} E_{\varphi_{\varepsilon}, \lambda, p_{\varepsilon}}\left(u_{\varepsilon}\right) .
$$

We claim that $u \in W^{1, \infty}(\Omega)$. To see this observe first that

$$
\int_{\Omega}\left|\nabla u_{\varepsilon}\right|^{p_{\varepsilon}} \leq p_{\varepsilon}\left(C+\left|\int_{\Omega} u_{\varepsilon}-\lambda \varphi_{\varepsilon} u_{\varepsilon}^{2}\right|\right) \leq p_{\varepsilon} C
$$

Therefore, using the semicontinuity of $u \mapsto \int|\nabla u|^{q}$ with respect to the weak convergence and Hölder inequality, we can compute for any fixed exponent $q$,

$$
\begin{aligned}
\int_{\Omega}|\nabla u|^{q} & \leq \liminf _{\varepsilon \rightarrow 0} \int_{\Omega}\left|\nabla u_{\varepsilon}\right|^{q} \\
& \leq \liminf _{\varepsilon \rightarrow 0}|\Omega|^{1-\frac{q}{p_{\varepsilon}}}\left(\int_{\Omega}\left|\nabla u_{\varepsilon}\right|^{p_{\varepsilon}}\right)^{\frac{q}{p_{\varepsilon}}} \\
& \leq \liminf _{\varepsilon \rightarrow 0}|\Omega|^{1-\frac{q}{p_{\varepsilon}}}\left(p_{\varepsilon} C\right)^{\frac{q}{p_{\varepsilon}}} \\
& =|\Omega| .
\end{aligned}
$$


The last inequality holds for any large $q$. Now taking $q \rightarrow+\infty$ yields

$$
\|\nabla u\|_{\infty}=\lim _{q \rightarrow+\infty}\left(\int_{\Omega}|\nabla u|^{q}\right)^{\frac{1}{q}} \leq \lim _{q \rightarrow+\infty}|\Omega|^{\frac{1}{q}} \leq 1
$$

which proves both that $u \in W^{1, \infty}(\Omega)$ and $\|\nabla u\|_{\infty} \leq 1$ (notice that $u \in L^{\infty}$ by the Sobolev inequality). Therefore, by definition of $E_{\varphi, \lambda}(u)$ we have that $E_{\varphi, \lambda}(u)=-\int_{\Omega} u+\lambda \int_{\Omega} \varphi u^{2}$ and passing to the liminf while $\varepsilon \rightarrow 0$ in $E_{\varphi_{\varepsilon}, \lambda, p_{\varepsilon}}\left(u_{p}\right)$ yields

$$
\begin{aligned}
\liminf _{\varepsilon \rightarrow 0} E_{\varphi_{\varepsilon}, \lambda, p_{\varepsilon}}\left(u_{\varepsilon}\right) & =\liminf _{\varepsilon \rightarrow 0}\left(\lambda \int_{\Omega} \varphi_{\varepsilon} u_{\varepsilon}^{2}-\int_{\Omega} u_{\varepsilon}+\frac{1}{p_{\varepsilon}} \int_{\Omega}\left|\nabla u_{\varepsilon}\right|^{p_{\varepsilon}}\right) \\
& =\liminf _{\varepsilon \rightarrow 0}\left(\frac{1}{p_{\varepsilon}} \int_{\Omega}\left|\nabla u_{\varepsilon}\right|^{p_{\varepsilon}}\right)-\int_{\Omega} u+\lambda \int_{\Omega} \varphi u^{2} \\
& \geq-\int_{\Omega} u+\lambda \int_{\Omega} \varphi u^{2}=E_{\varphi, \lambda}(u)
\end{aligned}
$$

which proves (6.3).

We will also need the following proposition.

Proposition 6.2. Let $K_{\lambda} \subseteq \Omega \subseteq \mathbb{R}^{2}$ be a sequence of convex sets such that $\chi_{K_{\lambda}}$ converges to $\chi_{K}$ in $L^{2}$. Then $K$ is also convex and denoting $u_{\lambda}$ the minimizer of $E_{\chi_{K_{\lambda}}, \lambda}$, we have that $u_{\lambda}(\mathbf{x})$ converges uniformly to $\mathbf{x} \mapsto \operatorname{dist}(\mathbf{x}, K)$ when $\lambda \rightarrow+\infty$.

Proof. Firstly notice that since the sets $K_{\lambda}$ are convex, the convergence of the characteristic functions in $L^{2}$ implies the convergence of $K_{\lambda}$ for the Hausdorff distance and in the sense of compacts, thus it follows that $K$ must be convex. To prove the proposition it is enough to prove that $E_{\lambda}:=E_{\chi_{K_{\lambda}, \lambda}}$ (as defined in (6.1)) $\Gamma$-converges in the uniform topology, to the functional

$$
E_{\infty}(u):=\left\{\begin{array}{cc}
-\int_{\Omega} u & \text { if } u \in W^{1, \infty}(\Omega), u=0 \text { a.e. on } K \text { and }\|\nabla u\|_{L^{\infty}} \leq 1 \\
+\infty & \text { otherwise. }
\end{array}\right.
$$

Indeed, the minimizer of $E_{\infty}$ is exactly $\mathbf{x} \mapsto \operatorname{dist}(\mathbf{x}, K)$.

The limsup inequality is easy, taking $u_{\lambda}:=u \chi_{\Omega \backslash K_{\lambda}}$ as a recovery sequence we have that $u_{\lambda}$ converges to $u$ in $L^{\infty}$ and

$$
E_{\lambda}(u)=-\int_{\Omega} u_{\lambda}
$$

thus taking the limsup we directly get

$$
\limsup _{\lambda} E_{\lambda}\left(u_{\lambda}\right)=E_{\infty}(u) .
$$

Now for the liminf inequality, assume that $u_{\lambda}$ is a sequence that converges uniformly to $u \in L^{\infty}$. Then since $K_{\lambda}$ converges to $K$ is Hausdorff distance, taking the limit in $\lambda \int_{K_{\lambda}} u_{\lambda}^{2}$, which is uniformly bounded in $\lambda$, it is easy to see that $u=0$ a.e. on $K$. Moreover by the uniform convergence we obtain that $u$ is Lipschitz and $\|\nabla u\|_{\infty} \leq 1$. Therefore taking the liminf in $E_{\lambda}\left(u_{\lambda}\right)$ we get the desired inequality that finishes the proof of the proposition.

Remark 6.3. We could change the convexity assumption in Proposition 6.2 into some different one like for instance uniformly Lipschitz domains, and still get the $\Gamma$-convergence result. 
Next we define the following functionals

$$
\mathcal{F}_{\varepsilon, \lambda}(\varphi):=(1-\varepsilon) \int_{\Omega} u_{\varphi, \lambda, \frac{1}{\varepsilon}} \mathrm{d} \mathbf{x}+\lambda_{1} \int_{\Omega} \varphi \mathrm{d} \mathbf{x}+\lambda_{2}\left(\int_{\Omega} \varepsilon|\nabla \varphi|^{2}+\frac{W(\varphi)}{\varepsilon} \mathrm{d} \mathbf{x}\right) .
$$

Where $W(t):=t^{2}(1-t)^{2}$ is the traditional double well potential involved in Modica and Mortola's functional. Then we finally denote the limiting functional in $\varepsilon$, defined on $L^{p_{0}}(\Omega)$ for $p_{0}>2$

$$
\mathcal{F}_{0, \lambda}(\varphi):=\left\{\begin{array}{cc}
\int_{\Omega} u_{K, \lambda}+\lambda_{1}|K|+\lambda_{2} \mathcal{H}^{1}(\partial K) & \text { if } \varphi \in B V(\Omega) \\
+\infty & \text { and } \varphi=\chi_{K} \text { for some } K \\
\text { Otherwise }
\end{array}\right.
$$

where $u_{K, \lambda}$ is defined as the minimizer for $E_{\chi_{K}, \lambda}$ (defined in (6.1)). Finally we define

$$
\mathcal{F}_{0, \infty}(\varphi):=\left\{\begin{array}{cc}
\int_{\Omega} \mathrm{d}(\mathbf{x}, K \cup \partial \Omega) \mathrm{d} \mathbf{x}+\lambda_{1}|K|+\lambda_{2} \mathcal{H}^{1}(\partial K) & \text { if } \varphi=\chi_{K}, K \text { convex } \\
+\infty & \text { Otherwise }
\end{array}\right.
$$

Theorem 6.4. Let $\lambda_{2} \neq 0$. Then we have the following diagram

$$
\mathcal{F}_{\varepsilon, \lambda} \underset{\varepsilon \rightarrow 0}{\stackrel{\Gamma}{\longrightarrow}} \mathcal{F}_{0, \lambda} \underset{\lambda \rightarrow+\infty}{\stackrel{\Gamma}{\longrightarrow}} \mathcal{F}_{0, \infty}
$$

for the $L^{2}$ topology, the second convergence holding under the extra assumption that $\mathcal{F}_{0, \lambda}$ is $+\infty$ if $K$ is not convex.

Remark 6.5. It is not clear wether one could let $\lambda$ depend on $\varepsilon$ and pass to the limit only a single time instead of letting first $\varepsilon \rightarrow 0$ and then $\lambda \rightarrow+\infty$, separately. Indeed, such a "uniform" $\Gamma$-convergence result would need the convergence of solutions $u_{\varphi_{\varepsilon}, \lambda_{\varepsilon}, p(\varepsilon)}$ to the solution $u_{\varphi, \infty, \infty}$. This convergence holds provided the rate of convergence of $\varphi_{\varepsilon}$ to $\varphi$ is fast enough compared to the convergence of $\lambda_{\varepsilon}$ to $+\infty$. Otherwise the $\Gamma$-limit, that can be characterized using capacitary measures, could be different from the one we would expect. It is therefore difficult to find a proper dependance of $\lambda$ with respect to $\varepsilon$ to make this convergence true for any $\varphi_{\varepsilon} \rightarrow \varphi$, that have a uniform bound of $\mathcal{F}_{\varepsilon}\left(\varphi_{\varepsilon}\right)$, say (this would be needed in the proof of the $\Gamma$-liminf). This is somehow not a real problem in view of numerical computations. Indeed, to approximate the average distance functional $\mathcal{F}_{0, \infty}$ one should first fix a very large $\lambda$ and then minimize $\mathcal{F}_{\varepsilon, \lambda}$ for some $\varepsilon$ small. Our convergence result guaranties that for $\varepsilon$ small enough, we will approximate $\mathcal{F}_{0, \infty}$, at least as much as $\mathcal{F}_{0, \lambda}$ does.

Remark 6.6. For the second convergence we need to restrict the class to convex sets only. This is not convenient for numerical computations because it is difficult to implement any topological or convexity constraint with the computer.

Proof of Theorem 6.4 (first convergence). We prove first the liminf inequality. Let $\varphi_{\varepsilon}$ be a sequence of functions converging to $\varphi$ in $L^{2}$. We want to prove that

$$
\mathcal{F}_{0}(\varphi) \leq \liminf _{\varepsilon \rightarrow 0} \mathcal{F}_{\varepsilon}\left(\varphi_{\varepsilon}\right)
$$

Without loss of generality we may assume that the liminf is finite and is a real limit. Since $\lambda_{2} \neq 0$ we know that $\int_{\Omega} \frac{W\left(\varphi_{\varepsilon}\right)}{\varepsilon}$ is bounded by a uniform constant, thus passing to the limit we deduce that $\int_{\Omega} W(\varphi)=0$ thus $\varphi=\chi_{K}$ a.e. for some set $K$. By [17] we already know $\varphi \in B V(\Omega)$ and that

$$
\liminf _{\varepsilon \rightarrow 0}\left(\int_{\Omega} \varepsilon\left|\nabla \varphi_{\varepsilon}\right|^{2}+\frac{W\left(\varphi_{\varepsilon}\right)}{\varepsilon}\right) \geq \mathcal{H}^{1}(\partial K)
$$

It is also clear that

$$
\int_{\Omega} \varphi_{\varepsilon} \rightarrow|K|
$$


Therefore to prove the liminf inequality it is enough to prove that

$$
\liminf _{\varepsilon \rightarrow 0}\left((1-\varepsilon) \int_{\Omega} u_{\varphi_{\varepsilon}, \lambda, p_{\varepsilon}}\right) \geq \int_{\Omega} u_{K, \lambda},
$$

where we set $p_{\varepsilon}:=1 / \varepsilon$ and $u_{K, \lambda}$ is the minimizer of $E_{\chi_{K}, \lambda}$ (as defined in (6.1)). But this is a direct consequence of Lemma 6.1, which precisely says that $u_{\varphi_{\varepsilon}, \lambda, p_{\varepsilon}}$ converges to $u_{K, \lambda}$ in $L^{2}$, yielding an equality in (6.6).

Let us now prove the limsup inequality. Let $\varphi=\chi_{K}$ be fixed. By Modica and Mortola's result and standard approximation by polygonal domains we know that there exists a sequence $\varphi_{\varepsilon}$ that converges to $\varphi$ in $L^{2}$ and such that

$$
\limsup _{\varepsilon \rightarrow 0}\left(\int_{\Omega} \varepsilon\left|\nabla \varphi_{\varepsilon}\right|^{2}+\frac{W\left(\varphi_{\varepsilon}\right)}{\varepsilon}\right) \leq \mathcal{H}^{1}(\partial K) .
$$

Since $\varphi_{\varepsilon}$ converges in $L^{2}$ it is clear that

$$
\int_{\Omega} \varphi_{\varepsilon} \rightarrow|K|
$$

Then it remains to prove the convergence of the $p$-compliance term, and this again directly comes from Proposition 6.1 as in the proof of the $\Gamma$-liminf.

Proof of Theorem 6.4 (second convergence). We prove first the liminf inequality. Let $\varphi_{\lambda}=\chi_{K_{\lambda}}$ be a sequence of characteristic functions converging to $\varphi=\chi_{K}$ in $L^{2}$, with $K_{\lambda}$ and $K$ convex. Then by convexity of $K_{\lambda}$ it is well known that the volume and perimeter are continuous with respect to this convergence. It is therefore enough to prove that

$$
\mathcal{F}(K) \leq \liminf _{\lambda \rightarrow+\infty} \int_{\Omega} u_{K_{\lambda}, \lambda}
$$

and this follows from Proposition 6.2.

The limsup also directly follows from Proposition 6.2 in an even weaker form, taking as a recovery sequence for a convex set $K$ the identically constant sequence $\chi_{K}$.

Acknowledgements. The authors wish to thank professor Giuseppe Buttazzo for fruitful discussions. They also would like to thank Jimmy Lamboley who has read a first version of this paper and gave to us precious remarks and corrections.

\section{REFERENCES}

[1] L. Ambrosio and C. Mantegazza, Curvature and distance function from a manifold. J. Geom. Anal. 8 (1998) $723-748$. Dedicated to the memory of Fred Almgren.

[2] L. Ambrosio and V.M. Tortorelli, Approximation of functionals depending on jumps by elliptic functionals via $\Gamma$-convergence. Comm. Pure Appl. Math. 43 (1990) 999-1036.

[3] D. Bucur, I. Fragalà and J. Lamboley, Optimal convex shapes for concave functionals. ESAIM: COCV (in press).

[4] G. Buttazzo and P. Guasoni, Shape optimization problems over classes of convex domains. J. Convex Anal. 4 (1997) $343-351$.

[5] G. Buttazzo and F. Santambrogio, Asymptotical compliance optimization for connected networks. Netw. Heterog. Media 2 (2007) 761-777 (electronic).

[6] G. Buttazzo and E. Stepanov, Optimal transportation networks as free Dirichlet regions for the Monge-Kantorovich problem. Ann. Scuola Norm. Super. Pisa Cl. Sci. 2 (2003) 631-678.

[7] G. Buttazzo, E. Oudet and E. Stepanov, Optimal transportation problems with free Dirichlet regions, in Variational methods for discontinuous structures, Progr. Nonlinear Differential Equations Appl. 51. Birkhäuser, Basel (2002) 41-65.

[8] G. Buttazzo, A. Pratelli, S. Solimini and E. Stepanov, Optimal urban networks via mass transportation, Lecture Notes in Mathematics 1961. Springer-Verlag, Berlin (2009).

[9] G. Buttazzo, E. Mainini and E. Stepanov, Stationary configurations for the average distance functional and related problems. Control Cybernet. 38 (2009) 1107-1130.

[10] M.C. Delfour and J.-P. Zolésio, Shape analysis via distance functions: local theory, in Boundaries, interfaces, and transitions (Banff, AB, 1995), CRM Proc. Lect. Notes 13. Amer. Math. Soc. Providence, RI (1998) 91-123.

[11] H. Federer, Curvature measures. Trans. Amer. Math. Soc. 93 (1959) 418-491.

[12] H. Federer, Geometric measure theory, Die Grundlehren der mathematischen Wissenschaften, Band 153. Springer-Verlag New York Inc., New York (1969). 
[13] A. Henrot and M. Pierre, Variation et optimisation de formes, Mathématiques 8 Applications (Berlin) [Mathematics ES Applications] 48. Springer, Berlin (2005). Une analyse géométrique [a geometric analysis].

[14] A. Lemenant, About the regularity of average distance minimizers in $\mathbb{R}^{2}$. J. Convex Anal. 18 (2011) $949-981$.

[15] A. Lemenant, A presentation of the average distance minimizing problem. Zap. Nauchn. Sem. S.-Petersburg. Otdel. Mat. Inst. Steklov. (POMI) 390 (2010) 117-146 (Proceedings of St. Petersburg Seminar, available online at http://www.pdmi.ras.ru/ znsl/2011/v390/abs117.html).

[16] C. Mantegazza and A. Mennucci, Hamilton-jacobi equations and distance functions on riemannian manifolds. Appl. Math. Optim. 47 (2003) 1-25.

[17] L. Modica and S. Mortola, Il limite nella $\Gamma$-convergenza di una famiglia di funzionali ellittici. Boll. Un. Mat. Ital. A 14 (1977) $526-529$.

[18] E. Paolini and E. Stepanov, Qualitative properties of maximum distance minimizers and average distance minimizers in $\mathbb{R}^{n}$. J. Math. Sci. (N. Y.) 122 (2004) 3290-3309. Problems in mathematical analysis.

[19] F. Santambrogio and P. Tilli, Blow-up of optimal sets in the irrigation problem. J. Geom. Anal. 15 (2005) 343-362.

[20] L. Simon, Lectures on geometric measure theory, Proceedings of the Centre for Mathematical Analysis 3. Australian National University, Australian National University Centre for Mathematical Analysis, Canberra (1983).

[21] E. Stepanov, Partial geometric regularity of some optimal connected transportation networks. J. Math. Sci. (N.Y.) 132 (2006) 522-552. Problems in mathematical analysis.

[22] P. Tilli, Some explicit examples of minimizers for the irrigation problem. J. Convex Anal. 17 (2010) 583-595. 